\title{
Design of the Spherical Interfaces under the EC Cryostat Stanchions
}

Engineering Note \# 3740.224-EN-282

Issued: February 14, 1991

Originator: Nicole Chlebos/Rick Luther 


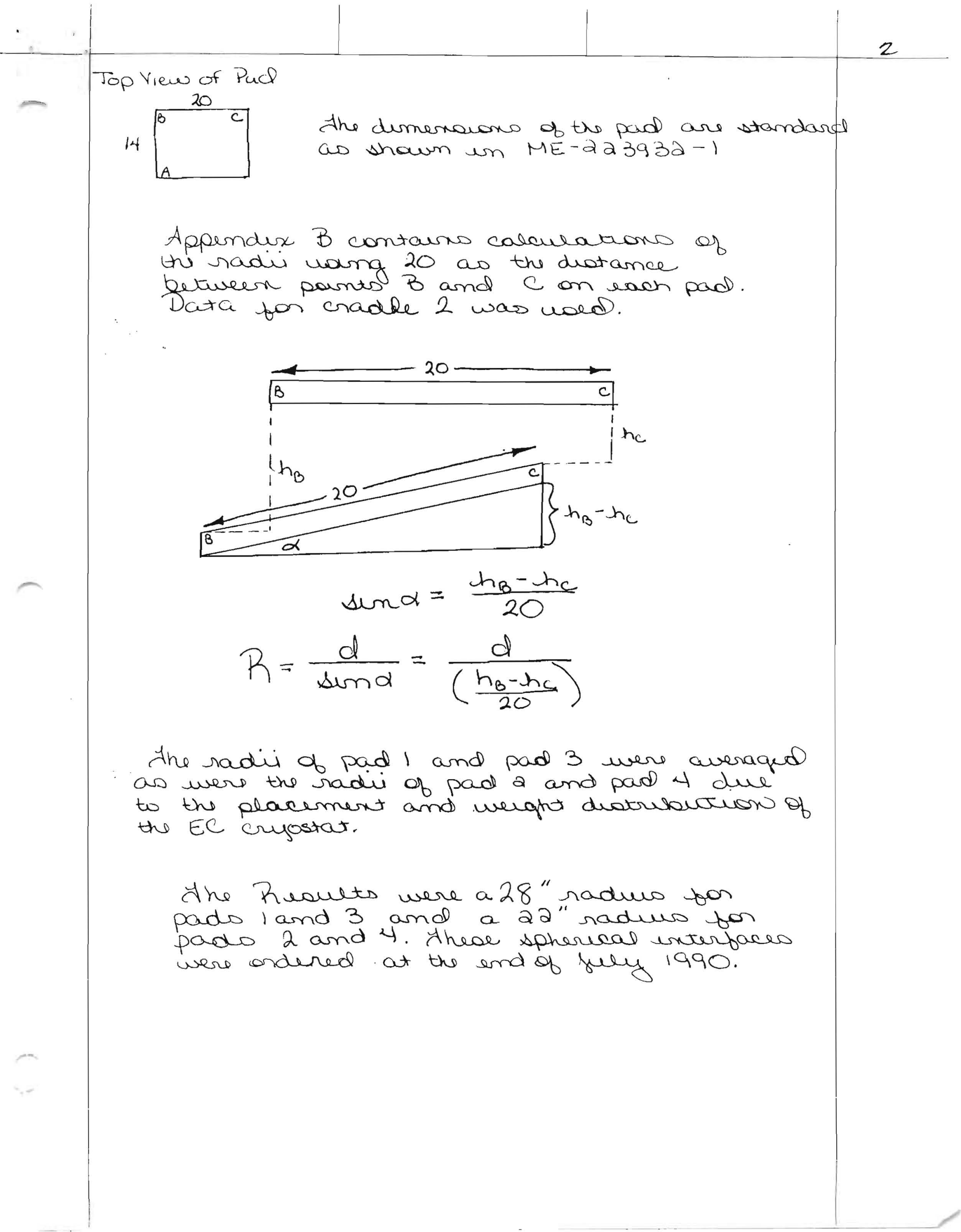


3

The distances betureer porto B and C for each pud were measured for each cradles. for Cradle 1, the average distance wow 17". ton Cradle 2, the average distance was 19".

Radii were recalculated en Appendix C young theses measurements.

The Radii for cradle 1 are scattered due to rigid body motion on torsion. the radius for cradle 2 wert down about an inch.

The 28 " and 22 " sphere cal interfaces were stull ordered based on the previous data so that the process was not delayed.

An analyses of points $A$ \& $B$ can not really be made for Pads $1+2$ and Pads Ht 4 due to the weight dep placement of the EC report. Pad 1 and 3 will aluaup deflect more than Pads 2 and I becallas the weight on pads 1 and 3 is three tames as much as pads 2 and 4

Appendix D contains the lest procedure And design calculations for thar ter fixtinse.

Appendix E contaniss plots of various deflections of the arms as taken from the survey result in CoppadixA. 
60 D-D CAAOLE LOAO TEGT O LAB A Appendixa

TD,RS CRADLE \# 1

a plan view of cradle only

BK $95-4$
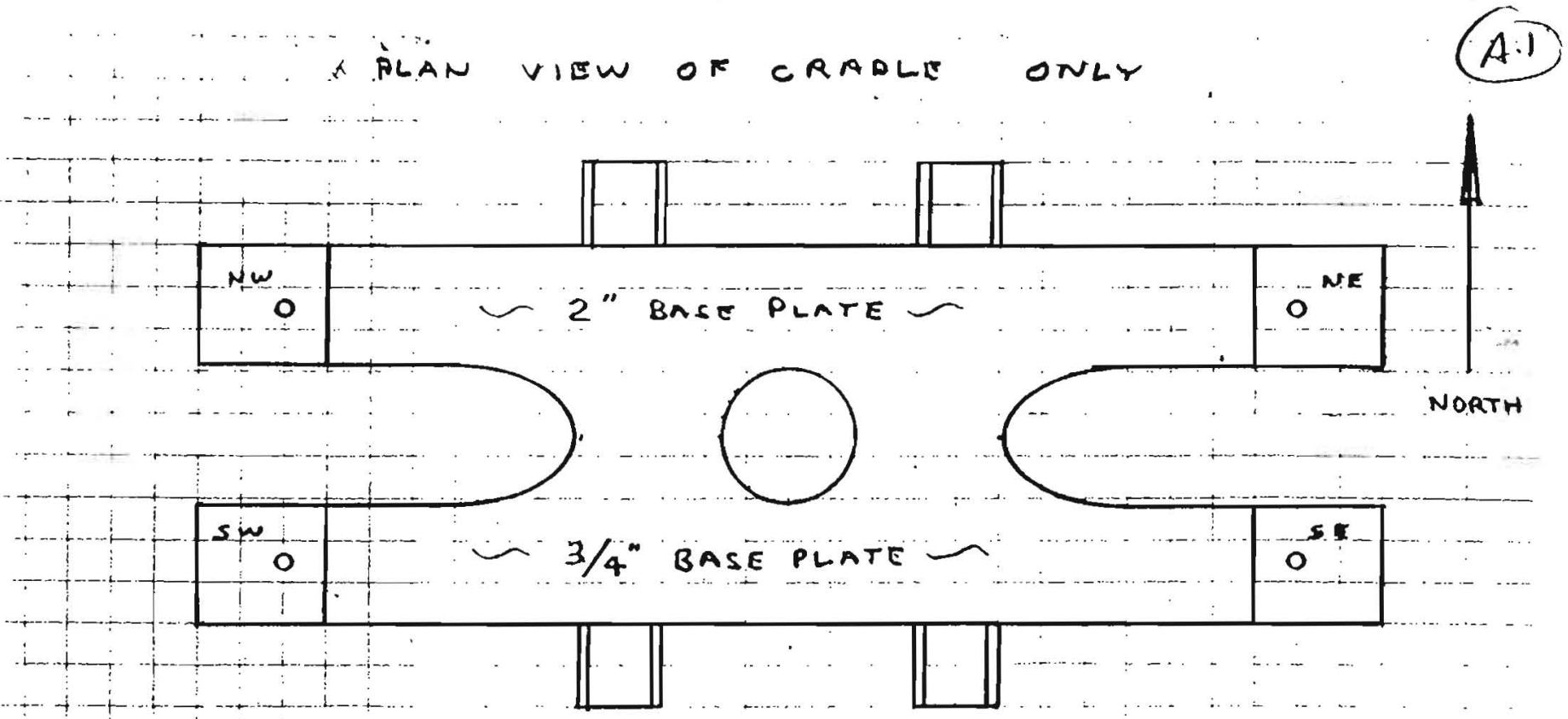

FRONT VIEW OF CRAOEE WITH LOAD TESTEA

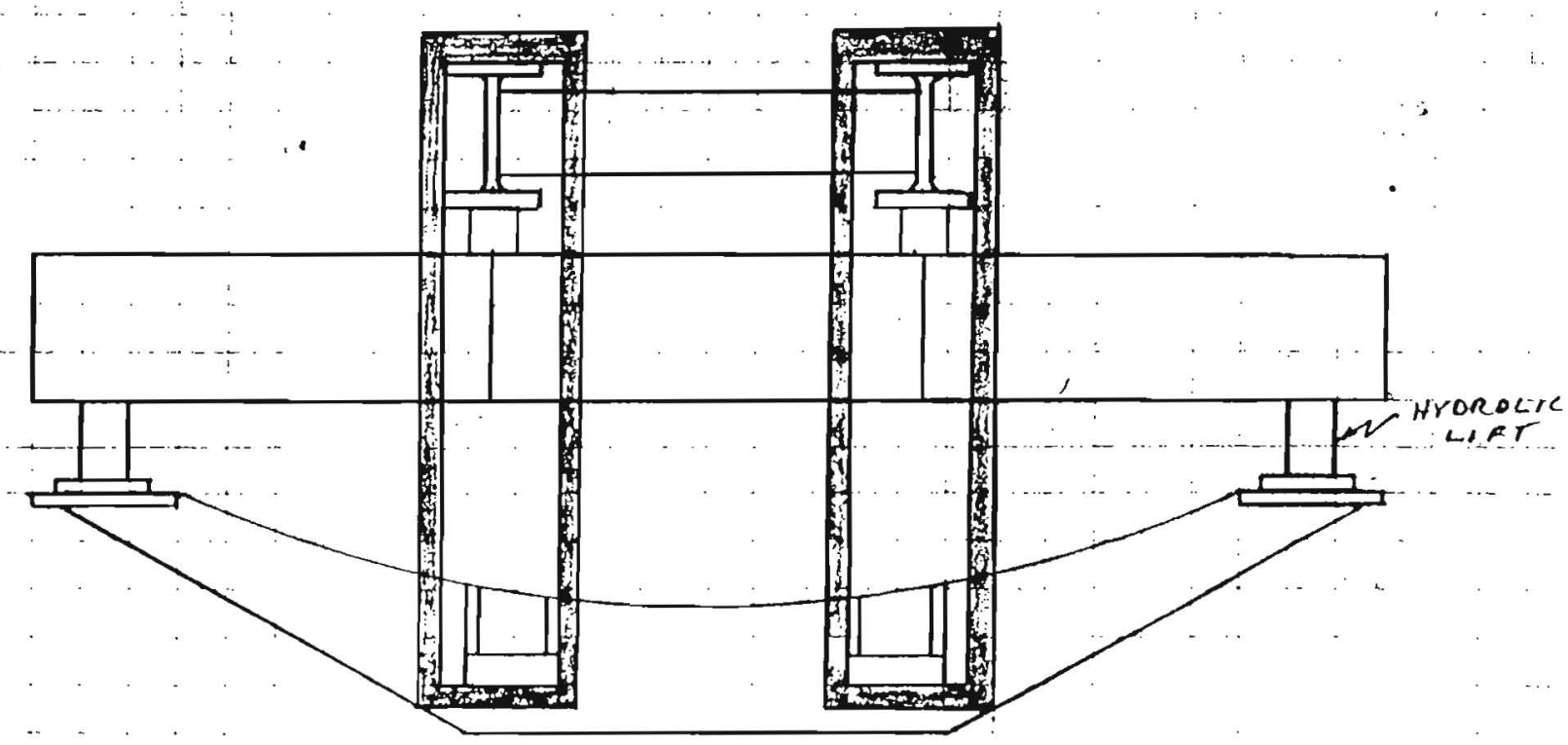




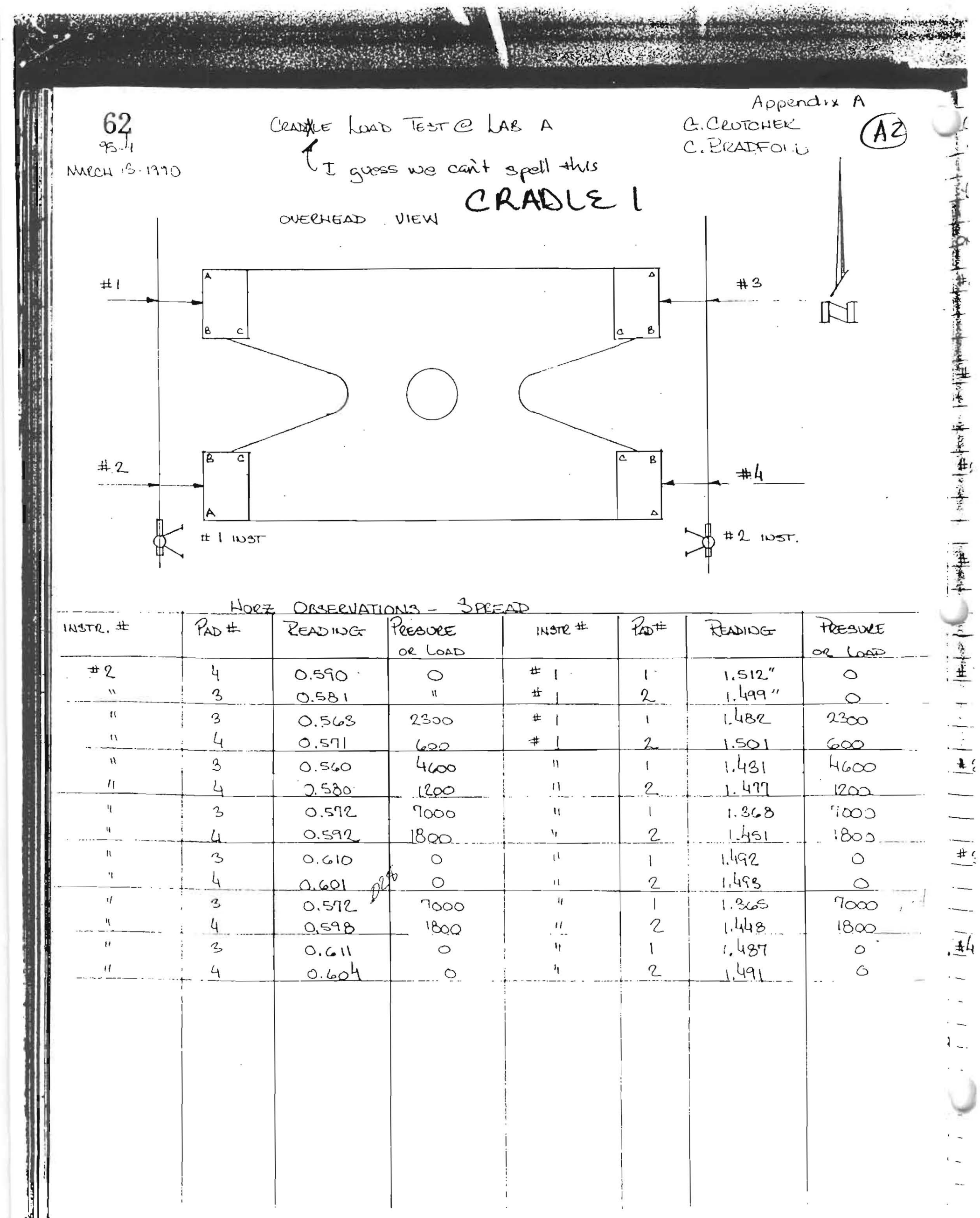




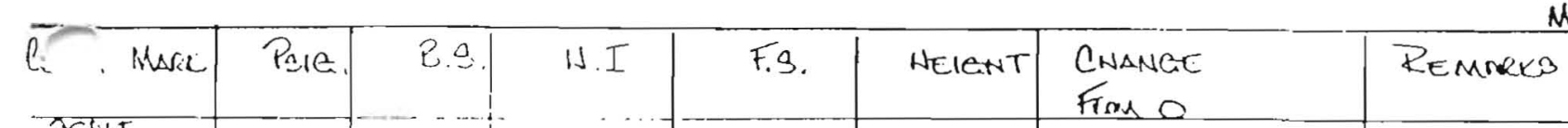
is 1 sctich 0 $\ddot{\imath}$

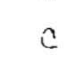

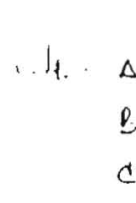
0 ×

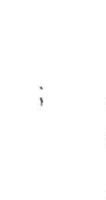
$=\frac{C}{\Delta} \begin{aligned} & \Delta \\ & B \\ & C\end{aligned}$ $=\because \frac{\Delta}{E}=$ *2. $a$
$e$
$c$ A e $c$ 2300

$B \quad 11$

11

600

- 11 " 


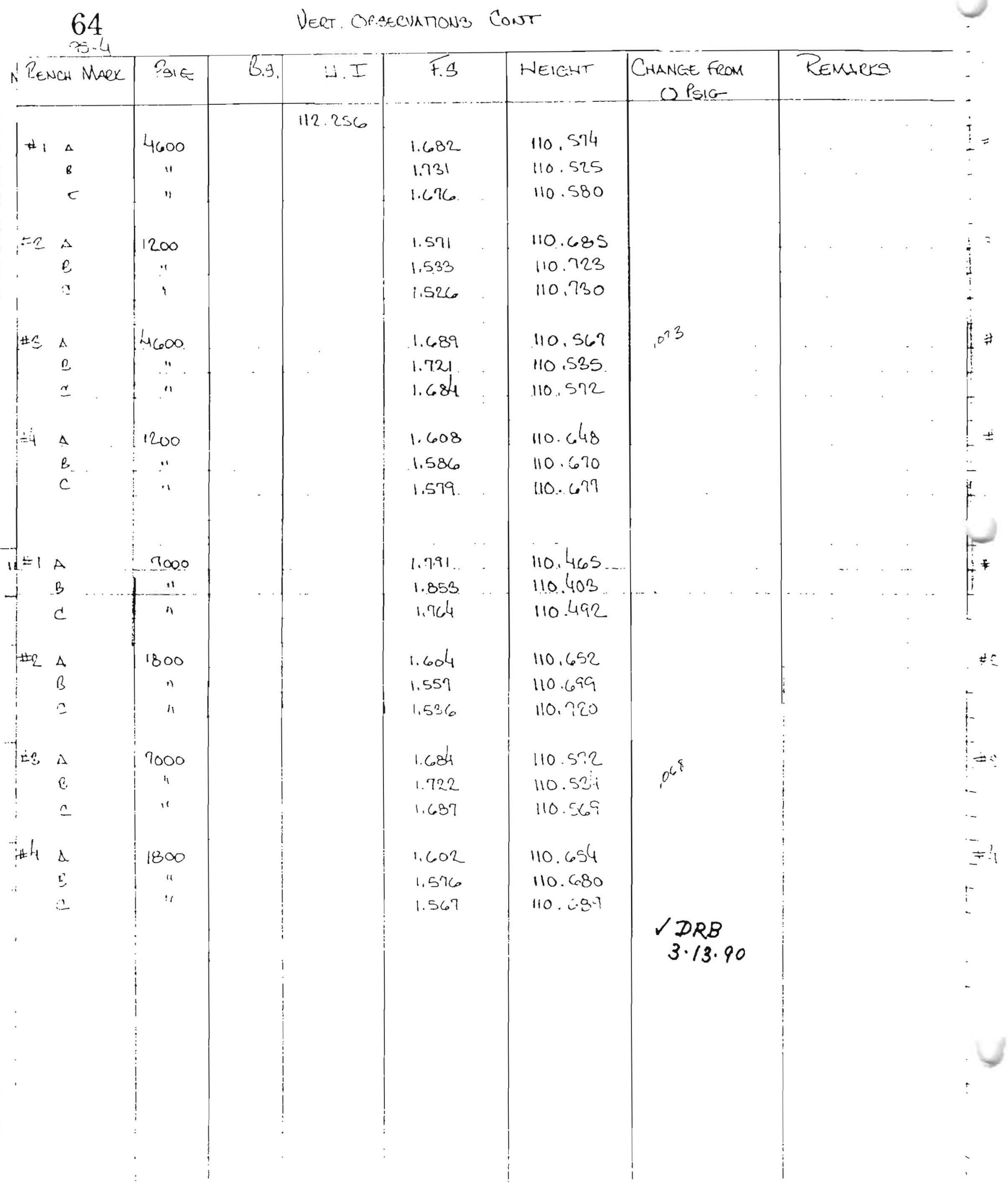




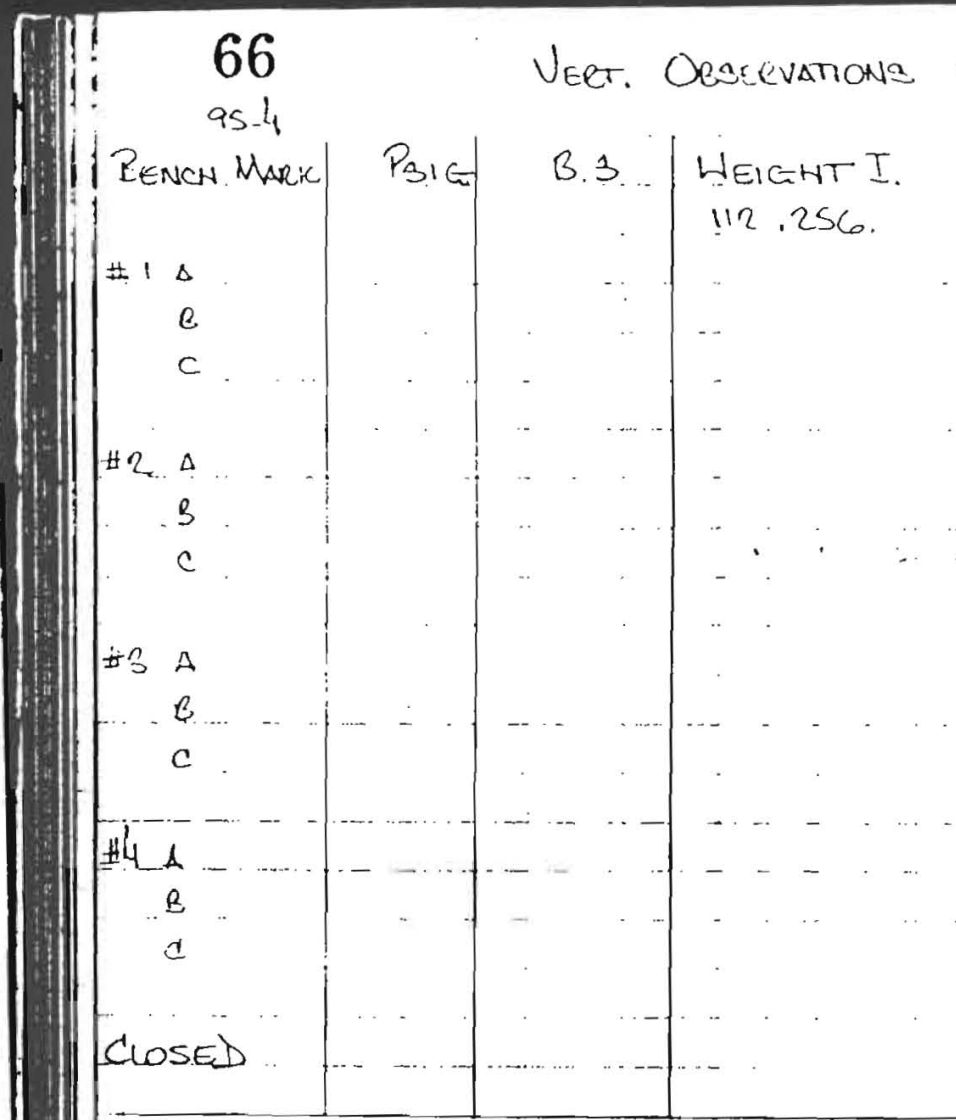

Cons.

ㄴ. Ceutcher

\section{as -4}

Vert. Oegrevamona Cont.

$\therefore$ Erdinono

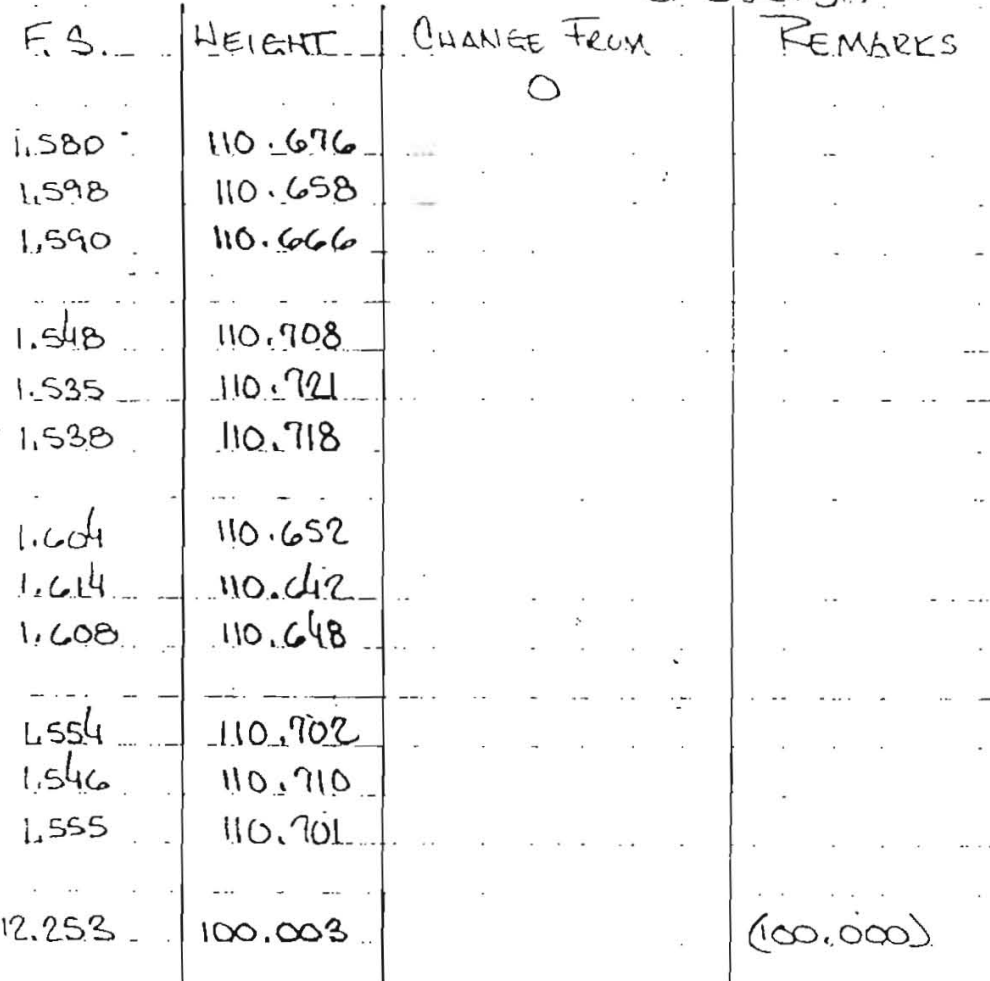




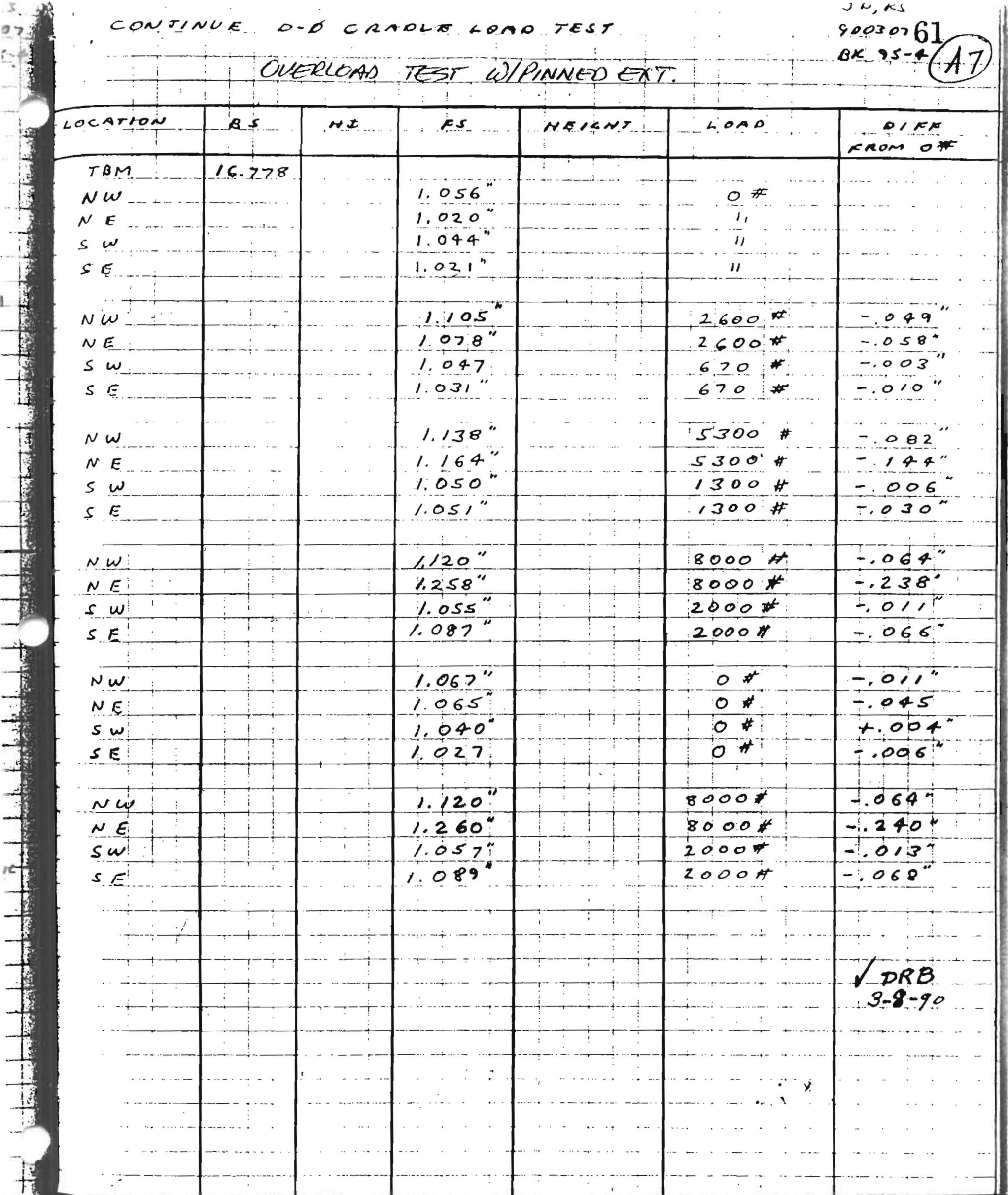




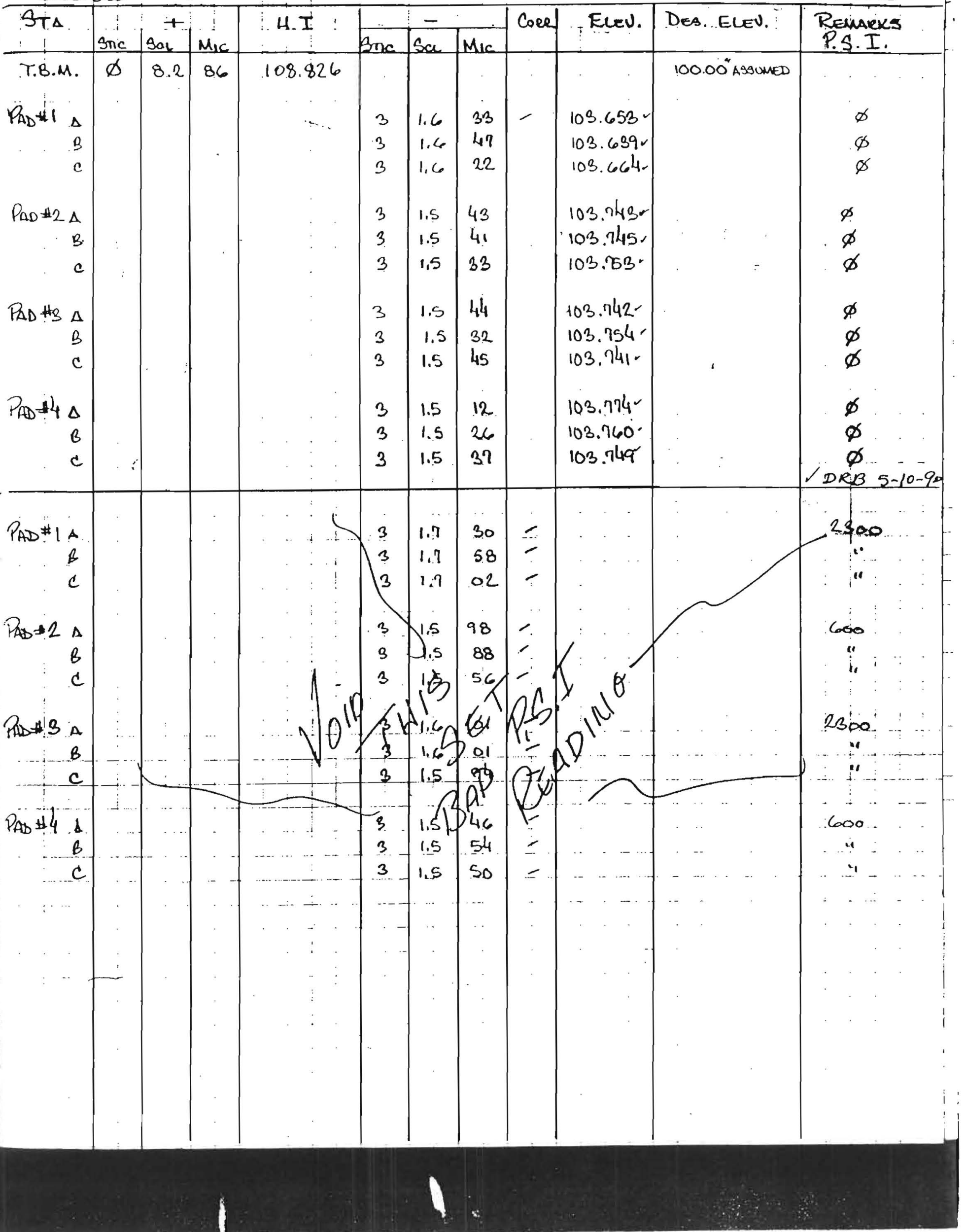




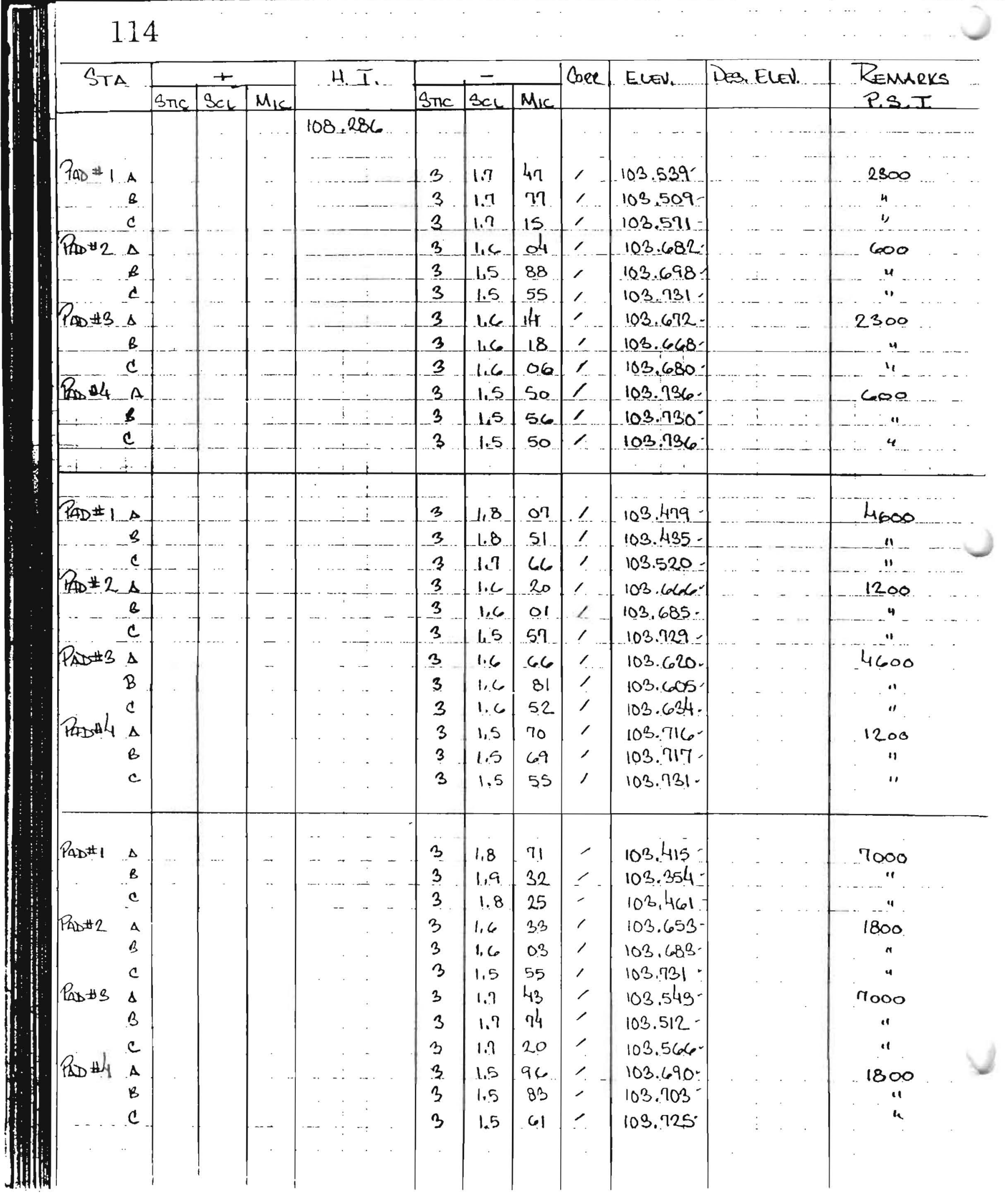



Sne Sas Mic

4.I:

\begin{tabular}{|l|l|l|l|}
\hline \multicolumn{2}{|c|}{-} & Core \\
\hline Snc & $S_{\text {Sal }}$ & Mic &
\end{tabular}

$E(E)$

Des. EleEl

PAD 2 A

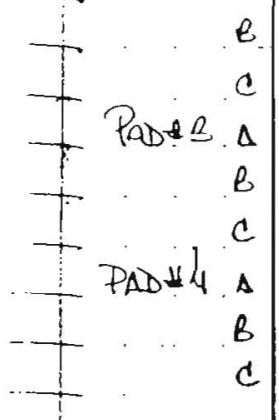

PQD $* 1$

$B$
$-C A$

Pa® $2 \Delta$

-.... B

$P A D+3 A$

A

c.

PaDd 4 A

108.286

3 1.6 $43, \quad 103.143$

$3.1 .658-103.628^{\circ}$

$31.632<103.654-$

3. 1.5 45 $; 103.941-$

$3-1.542=103.744$

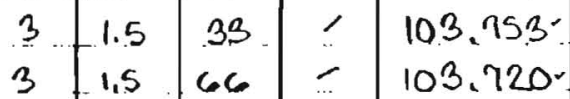

$3 \quad 1.557=103.729$.

$31.562=103.724$.

$31.511=103.775-$

$3 \quad 1.523=103.763^{\circ}$

$31.533=109.953$

3. $1.857=103.429$

$31.91 .8-103.368$.

$3 \quad 1.8 \quad 15-103.4 \pi=$

$3-1.6-32=103.654$.

$3-1.6$ ol 103.685

$3 \quad 1.5 .53=103.733$.

$3-1.956-103.530=$

$3 \quad 1.7 \quad 87 / 103,499$.

$3 \quad 129-103.557=$

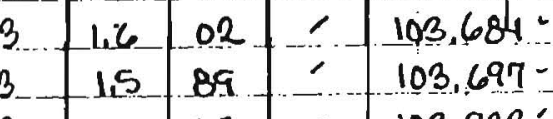

$3 \quad 1.563=103.723^{\circ}$

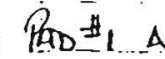

$\therefore-1-1$

3 1.6

13. 108.643 .

$3-1.6-59=103.627$.

$31.631=103.655$.

Pad $+2 A$

$3.1 .5 \quad 45$

$103.741^{-}$

$3 \quad 15 \quad 41-103.745$.

$3 \quad 1.5-32=103.754$

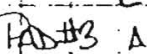

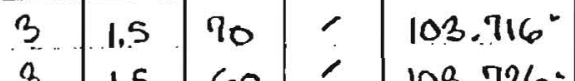

$\begin{array}{lll}3 & 1.5 & 60 \\ 3 & 1.5 & 65=109.726: \\ 3 & 1.5 & 11\end{array}$

$31.511=103.775$.

PADHA A

$3 \quad 1.523=103.763^{\circ}$

$31.532=103.954$.

$\begin{array}{lll}\phi & 8.2 \quad 85\end{array}$

$100.001-(100.000)$

$\checkmark D R B$

$5-10-90$ 


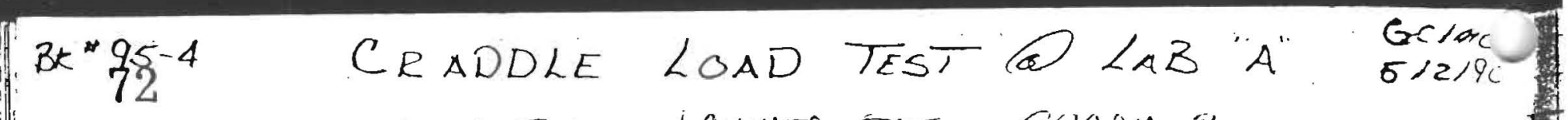

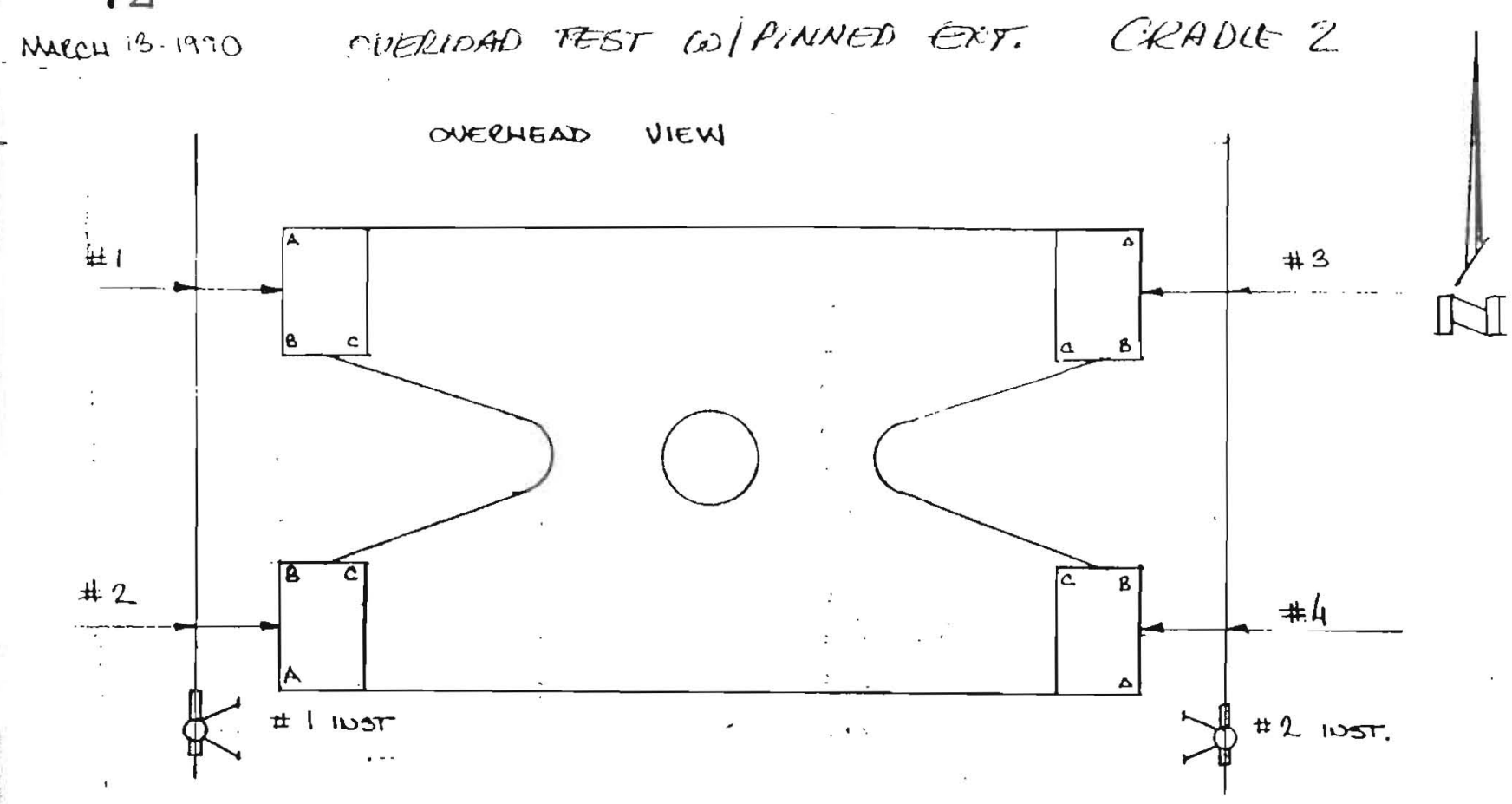

HORZ OBSERVATONAS - SPREAD:

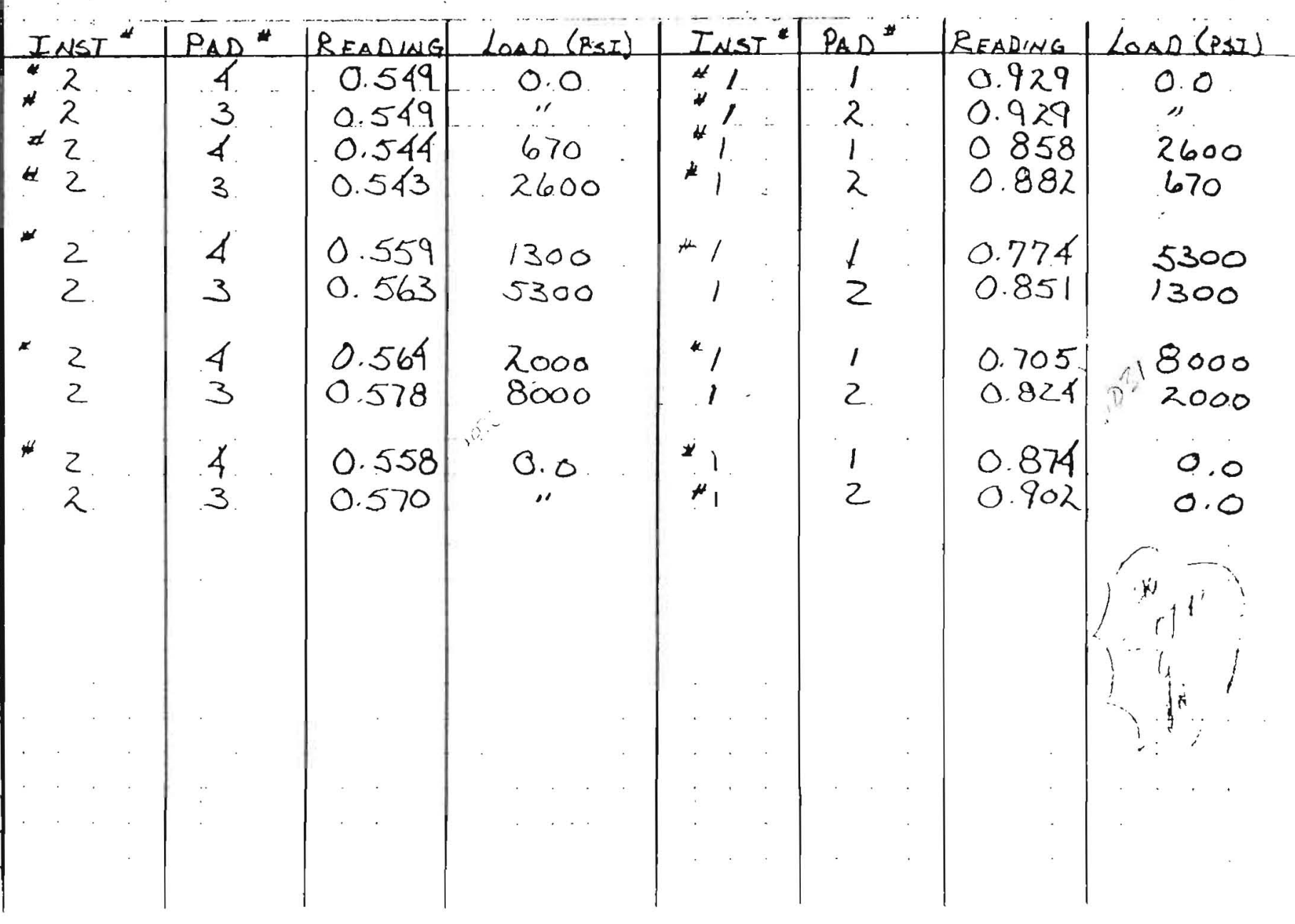




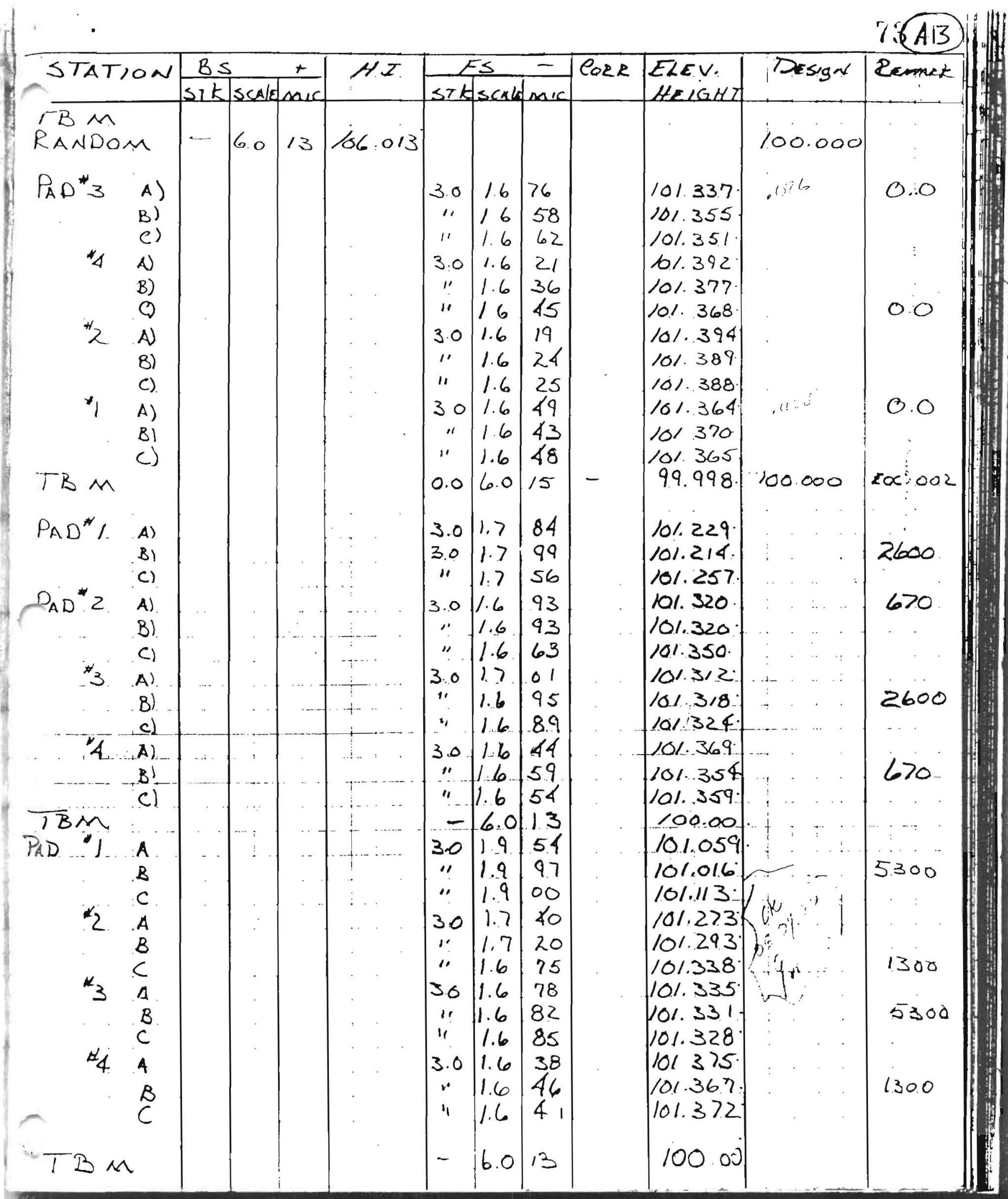


74

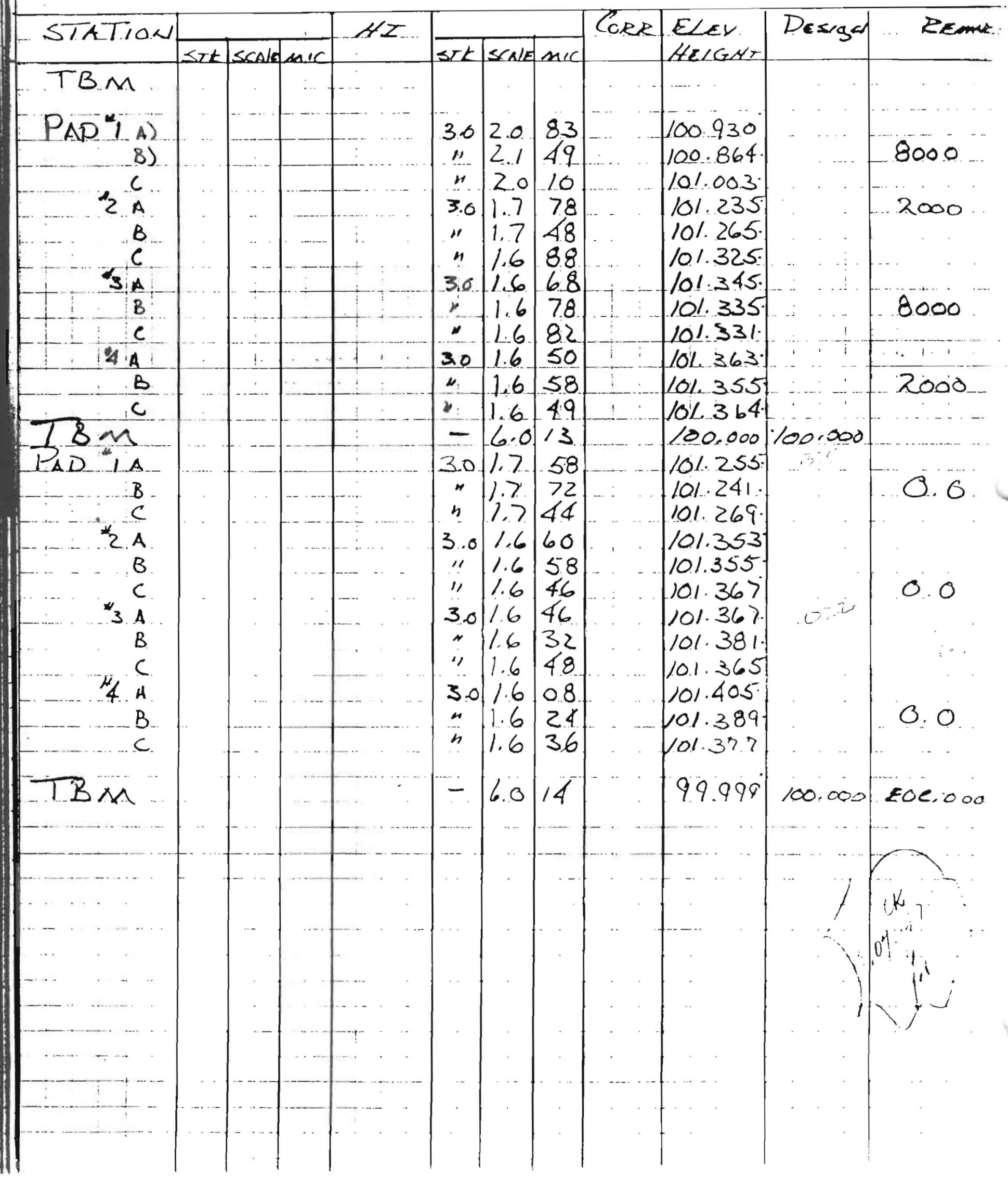




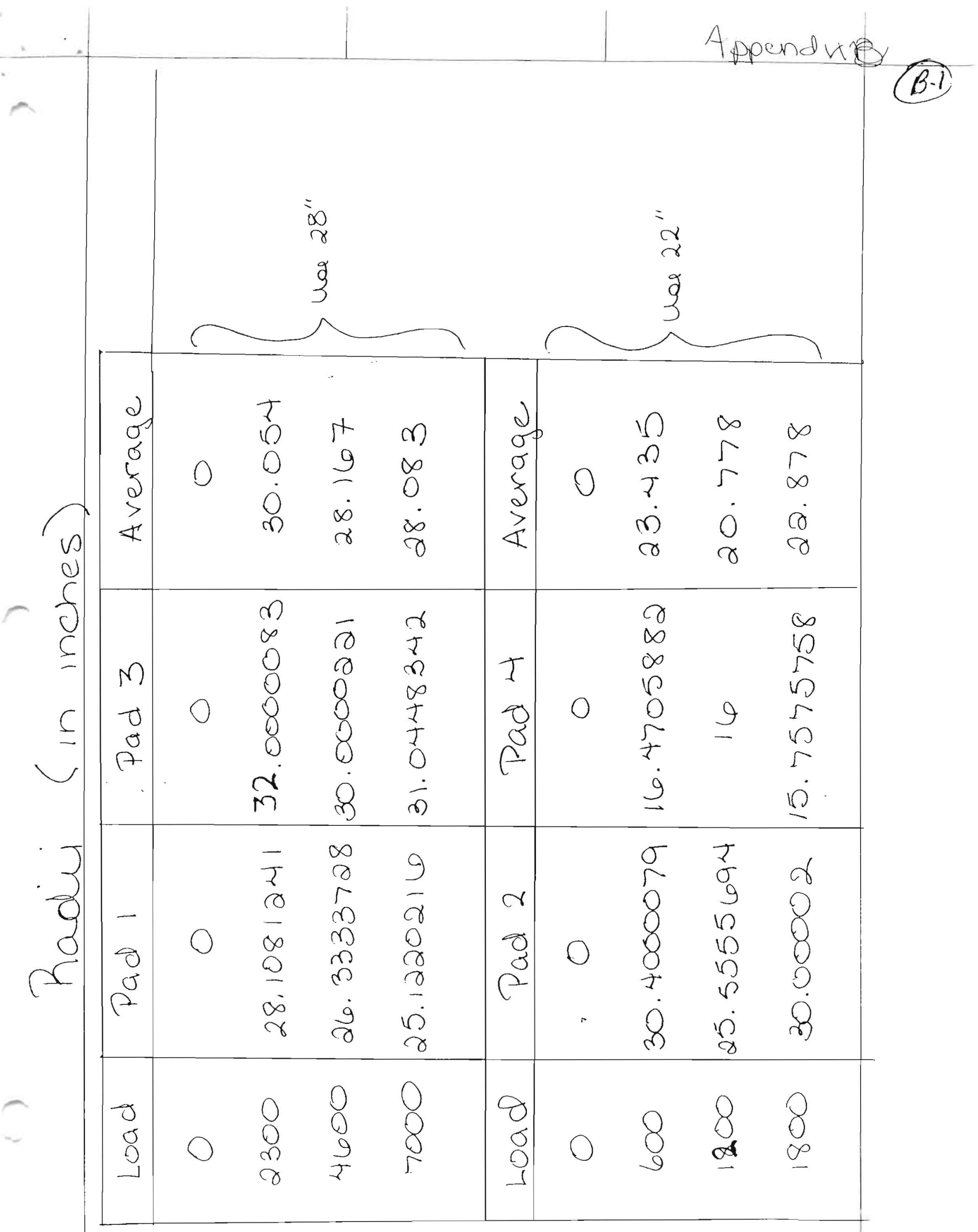




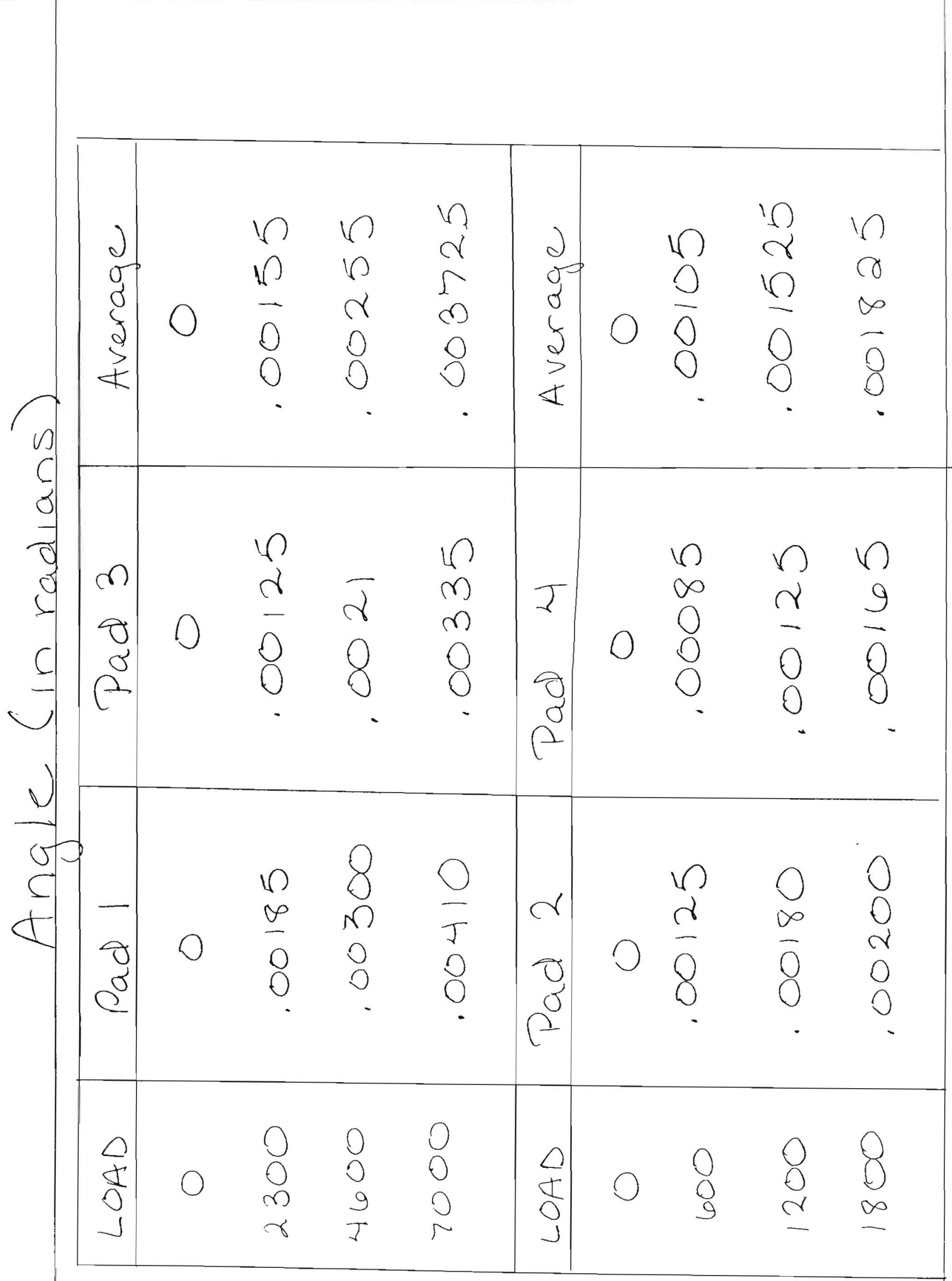


Pts $B$ and $C$

$P A D$

\begin{tabular}{|c|c|c|c|c|c|c|}
\hline & A & $\mathrm{B}$ & C & D & E & $\mathbf{F}$ \\
\hline 1 & Load psi & Vert Disp. B & Vert Disp. C & Sine of Angle & Horz Disp. & Radius \\
\hline 2 & 0 & 0 & 0 & 0 & 0 & \#DIV/0! \\
\hline 3 & 2300 & 0.13 & 0.093 & 0.00185 & 0.052 & 28.1081241 \\
\hline 4 & 4600 & 0.204 & 0.144 & 0.003 & 0.079 & 26.3333728 \\
\hline 5 & 7000 & 0.285 & 0.203 & 0.0041 & 0.103 & 25.1220216 \\
\hline 6 & $P A D 2$ & & & & & \\
\hline 7 & Load Psi & Vert Disp. B & Vert Disp. C & Sine of Angle & Horz Disp. & Radius \\
\hline 8 & 100 & 0 & 0 & 0 & 0 & \#DIV/O! \\
\hline 9 & 600 & 0.047 & 0.022 & 0.00125 & 0.038 & 30.4000079 \\
\hline 10 & 1200 & 0.06 & 0.024 & 0.0018 & 0.046 & 25.5555694 \\
\hline 11 & 1800 & 0.062 & 0.022 & 0.002 & 0.06 & 30.00002 \\
\hline 12 & PAD 3 & & & & & \\
\hline 13 & Load osi & Vert Disp. B & Vert Disp. C & Sine of Angle & Horz Disp. & Radius \\
\hline 14 & 10 & 0 & 0 & 0 & 0 & \#DIV/0! \\
\hline 15 & 2300 & 0.086 & 0.061 & 0.00125 & 0.04 & 32.0000083 \\
\hline 16 & 4600 & 0.149 & 0.107 & 0.0021 & 0.063 & 30.0000221 \\
\hline 17 & 7000 & 0.242 & 0.175 & 0.00334999 & 0.104 & 31.0448342 \\
\hline 18 & $P A D$ 4 & & & & & \\
\hline 19 & Load osi & Vert Disp. B & Vert Disp.C & Sine of Angle & Horz Disp. & Radius \\
\hline 20 & 0 & 0 & 0 & 0 & 0 & \#DIV/0! \\
\hline 21 & 600 & 0.03 & 0.013 & 0.00085 & 0.014 & 16.4705882 \\
\hline 22 & 1200 & 0.043 & 0.018 & 0.00125 & 0.02 & 16 \\
\hline 23 & 1800 & 0.057 & 0.024 & 0.00165 & 0.026 & 15.7575758 \\
\hline
\end{tabular}

Vert Disp. = Reading at Load $\varnothing$ - Reading at hoad Given Horz Disp = Horz Beading at Load $\varnothing$ - Beading at given Load Angle $=\mid$ (Vert. Disp. B - Vert. Disp C ) $\mid / 2 \phi$ Sine of Angle = Angle (when angle is in radians) Radius $=$ Horz. Disp./Sine of Angle

* 20 is the given length of the pad from ME-223932-1 
Pts $B$ and $C$

\begin{tabular}{|c|c|c|c|}
\hline & $\mathrm{G}$ & $A n q$ & 10 \\
\hline 1 & Angle (in rad & 1) $(\ln \cdot \operatorname{de})$ & \\
\hline 2 & 0 & 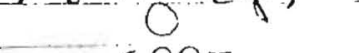 & 7 \\
\hline 3 & 0.00185 & .105997 & 2.300 \\
\hline 4 & 0.003 & .1718868 & 4600 \\
\hline 5 & 0.00409999 & .2349113 & 2000 \\
\hline 6 & 1)A & $D 2 \ldots$ & \\
\hline 7 & Angle (in rad) & & \\
\hline 8 & 0 & 0 & \\
\hline 9 & 0.00125 & .0716196 & \\
\hline 10 & 0.0018 & .1031322 & \\
\hline 11 & 0.002 & .1145914 & \\
\hline 12 & $P A$ & $A D 3$ & \\
\hline 13 & Angle (in rad) & & \\
\hline 14 & $\begin{array}{r}0 \\
\end{array}$ & 0 & \\
\hline 15 & 0.00125 & .0716196 & $\hat{\alpha}$ \\
\hline 16 & 0.0021 & .1203209 & \\
\hline 17 & 0.00335 & $.19,9401$ & \\
\hline 18 & $P A$ & 504 & \\
\hline 19 & Angle (in rad & 1) & \\
\hline 20 & 0 & 0 & 0 \\
\hline 21 & 0.00085 & .0487014 & 600 \\
\hline 22 & 0.00125 & $.0716 / 96$ & 1200 \\
\hline 23 & 0.00165 & 1.0945379 & 180 \\
\hline
\end{tabular}


Pts $A$ and $B$

\begin{tabular}{|c|c|c|c|c|c|}
\hline & A & B & C & D & $\mathrm{E}$ \\
\hline 1 & Load as-i & Vert Disp. A & Vert Disp. B & Angle (rad) & Horz Disp. \\
\hline 2 & 100 & 0 & 0 & 0 & 0 \\
\hline 3 & 2300 & 0.114 & 0.13 & 0.00114286 & 0.052 \\
\hline 4 & 4600 & 0.174 & 0.204 & 0.00214286 & 0.079 \\
\hline 5 & 7000 & 0.238 & 0.285 & 0.00335714 & 0.103 \\
\hline 6 & PAD 2 & & & & \\
\hline 7 & Load Osi & Vert Disp. A & Vert Disp. B & Angle ( $\mathrm{rac})$ & Horz Disp. \\
\hline 8 & 0 & 0 & 0 & 0 & 0 \\
\hline 9 & 600 & 0.061 & 0.047 & 0.001 & 0.038 \\
\hline 10 & 1200 & 0.077 & 0.06 & 0.00121429 & 0.046 \\
\hline 11 & 1800 & 0.09 & 0.062 & 0.002 & 0.06 \\
\hline 12 & PAD 3 & & & & \\
\hline 13 & Load psi & Vert Disp. A & Vert Disp. $B$ & Angle (rad)) & Horz Disp. \\
\hline 14 & 10 & 0 & 0 & 0 & 0 \\
\hline 15 & 2300 & 0.07 & 0.086 & 0.00114286 & 0.04 \\
\hline 16 & 4600 & 0.122 & 0.149 & 0.00192857 & 0.063 \\
\hline 17 & 7000 & 0.199 & 0.242 & 0.00307143 & 0.104 \\
\hline 18 & PAD y & & & & \\
\hline 19 & Load psic & Vert Disp. A & Vert Disp. B & Horz Disp. & Angle (rad) \\
\hline 20 & 0 & 0 & $\ulcorner 0$ & 0 & 0 \\
\hline 21 & 600 & 0.038 & 0.03 & 0.014 & 0.00057143 \\
\hline 22 & 1200 & 0.058 & 0.043 & 0.02 & 0.00107143 \\
\hline 23 & 1800 & 0.084 & 0.057 & 0.026 & 0.00192857 \\
\hline
\end{tabular}

Angle $=\left(\right.$ Vert Disp B-Vert. Disp.A) $/ 14^{*}$

* I4 is the given width of the pacl from ME - 223932-1 


\begin{tabular}{|c|c|c|c|c|c|c|}
\hline & A & B & C & D & $E$ & $\mathrm{~F}$ \\
\hline 1 & LOAD & VERT DISP B & VERT DISP C & SIN OF ANGLE & HORZ DISP. & RADIUS \\
\hline 2 & & & & & & \\
\hline 3 & Pad 1 & Pad 1 & Pad 1 & Pad 1 & Pad 1 & Pad 1 \\
\hline 4 & 0 & 0 & 0 & 0 & 0 & \#DIV/0! \\
\hline 5 & 2300 & 0.066 & 0.043 & 0.00135294 & 0.03 & 22.173913 \\
\hline 6 & 4600 & 0.173 & 0.116 & 0.00335294 & 0.081 & 24.1578947 \\
\hline 7 & 7000 & 0.295 & 0.204 & 0.00535294 & 0.144 & 26.9010989 \\
\hline 8 & Pad 2 & Pad 2 & Pad 2 & Pad 2 & Pad 2 & Pad 2 \\
\hline 9 & 0 & 0 & 0 & 0 & 0 & \#DIV/O! \\
\hline 10 & 600 & -0.009 & -0.012 & 0.00017647 & -0.002 & -11.333333 \\
\hline 11 & 1200 & 0.01 & -0.004 & 0.00082353 & 0.022 & 26.7142857 \\
\hline 12 & 1800 & 0.034 & 0.006 & 0.00164706 & 0.048 & 29.1428571 \\
\hline 13 & Pad 3 & Pad 3 & Pad 3 & Pad 3 & Pad 3 & Pad 3 \\
\hline 14 & 0 & 0 & 0 & 0 & 0 & \#DIV/0! \\
\hline 15 & 2300 & 0.083 & 0.06 & 0.00135294 & 0.018 & 13.3043478 \\
\hline 16 & 4600 & 0.093 & 0.067 & 0.00152941 & 0.021 & 13.7307692 \\
\hline 17 & 7000 & 0.094 & 0.07 & 0.00141176 & 0.009 & 6.375 \\
\hline 18 & Pad 4 & Pad 4 & Pad 4 & Pad 4 & Pad 4 & Pad 4 \\
\hline 19 & 0 & 0 & 0 & 0 & 0 & \#DIV/0! \\
\hline 20 & 600 & 0.024 & 0.016 & 0.00047059 & 0.019 & 40.375 \\
\hline 21 & 1200 & 0.029 & 0.018 & 0.00064706 & 0.01 & 15.4545455 \\
\hline 22 & 1800 & 0.019 & 0.006 & 0.00076471 & -0.002 & -2.6153846 \\
\hline
\end{tabular}

\begin{tabular}{|c|c|c|c|}
\hline$\angle O A D$ & PAD I Radius & Pad 3 Radius & Average Radius \\
\hline 0 & 0 & 0 & 0 \\
\hline 2300 & 22.174 & 13.304 & 17.739 \\
\hline 4600 & 24.158 & 13.731 & 18.9445 \\
\hline 7000 & 26.901 & 6.375 & 16.6 .38 \\
\hline$\angle O A D$ & Pad a Radius & Pad \& Radius & Average Radius \\
\hline 0 & 0 & 0 & 0 \\
\hline 600 & -11.333 & 40.375 & 14.521 \\
\hline 1200 & 26.714 & 15.455 & 21.0845 \\
\hline 1800 & 24.143 & -2.615 & 13.264 \\
\hline & & & \\
\hline
\end{tabular}




\begin{tabular}{|c|c|c|c|c|c|c|}
\hline & $\mathbf{G}$ & $\begin{array}{r}\mathbf{H} \\
\end{array}$ & I & $\mathbf{J}$ & K & $\mathbf{L}$ \\
\hline 1 & ANGLE & HORZ READING & VERT READ A & VERT READ B & VERT READ C & \\
\hline 2 & & & & & & \\
\hline 3 & Pad 1 & Pad 1 & Pad 1 & Pad 1 & Pad 1 & Pad 1 \\
\hline 4 & 0 & 1.512 & 110.712 & 110.698 & 110.696 & \\
\hline 5 & 0.00135294 & 1.482 & 110.667 & 110.632 & 110.653 & \\
\hline 6 & 0.00335294 & 1.431 & 110.574 & 110.525 & 110.58 & \\
\hline 7 & 0.00535294 & 1.368 & 110.465 & 110.403 & 110.492 & \\
\hline 8 & Pad 2 & Pad 2 & Pad 2 & Pad 2 & Pad 2 & Pad 2 \\
\hline 9 & 0 & 1.499 & \begin{tabular}{|l|}
110.725 \\
\end{tabular} & 110.733 & 110.726 & \\
\hline 10 & 0.00017647 & 1.501 & 110.716 & 110.742 & 110.738 & \\
\hline 11 & 0.00082353 & 1.477 & 110.685 & 110.723 & 110.73 & \\
\hline 12 & 0.00164706 & 1.451 & 110.652 & 110.699 & 110.72 & \\
\hline 13 & Pad 3 & Pad 3 & Pad 3 & Pad 3 & Pad 3 & Pad 3 \\
\hline 14 & 0 & 0.581 & 110.64 & 110.628 & 110.639 & \\
\hline 15 & 0.00135294 & 0.563 & 110.57 & 110.545 & 110.579 & \\
\hline 16 & 0.00152941 & 0.56 & 110.567 & 110.535 & 110.572 & \\
\hline 17 & 0.00141176 & 0.572 & 110.572 & 110.534 & 110.569 & \\
\hline 18 & Pad 4 & Pad 4 & Pad 4 & Pad 4 & Pad 4 & Pad 4 \\
\hline 19 & 0 & 0.59 & 110.689 & 110.699 & \begin{tabular}{|r|}
110.695 \\
\end{tabular} & \\
\hline 20 & 0.00047059 & 0.571 & 110.658 & 110.675 & 110.679 & \\
\hline 21 & 0.00064706 & 0.58 & 110.648 & 110.67 & 110.677 & \\
\hline 22 & 0.00076471 & 0.592 & 110.654 & 110.68 & 110.689 & \\
\hline
\end{tabular}

\begin{tabular}{|c|c|c|c|}
\hline Load & Pad I Angle & Pad 3 Angle & Average Angle \\
\hline 0 & 0 & 0 & 0 \\
\hline 2300 & .001353 & .001353 & .001353 \\
\hline 4600 & .003353 & .001529 & .002441 \\
\hline 7000 & .005353 & .001412 & .003383 \\
\hline Load & Pad 2 Angle & Pad -1 Angle & Average Angle \\
\hline 0 & 0 & 0 & 0 \\
\hline 600 & .0001765 & .0004706 & .0003235 \\
\hline 1200 & .0008235 & .0006471 & .0007353 \\
\hline 1800 & .001647 & .0007647 & .0012058 \\
\hline & & & \\
\hline
\end{tabular}

Page 2 
CRADE2

Pts $B$ and $C$

\begin{tabular}{|c|c|c|c|c|c|c|}
\hline & A & B & C & D & $E$ & $\mathrm{~F}$ \\
\hline 1 & Load & Vert Disp. & Vert Disp. & Sine of Angle & Horz Disp. & Radius \\
\hline 2 & 0 & 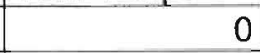 & 0 & 0 & 0 & \#DIV/0! \\
\hline 3 & 2300 & 0.13 & 0.093 & 0.00194737 & 0.052 & 26.7027196 \\
\hline 4 & 4600 & 0.204 & 0.144 & 0.00315789 & 0.079 & 25.0167082 \\
\hline 5 & 7000 & 0.285 & 0.203 & 0.00431579 & 0.103 & 23.8659277 \\
\hline 6 & & & & & & \\
\hline 7 & Load & Vert Disp. & Vert Disp. & Sine of Angle & Horz Disp. & Radius \\
\hline 8 & 0 & 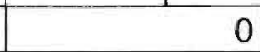 & 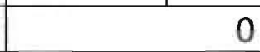 & 0 & 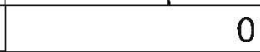 & \#DIV/O! \\
\hline 9 & 600 & 0.047 & 0.022 & 0.00131579 & 0.038 & 28.8800083 \\
\hline 10 & 1200 & 0.06 & 0.024 & 0.00189474 & 0.046 & 24.2777923 \\
\hline 11 & 1800 & 0.062 & 0.022 & 0.00210526 & 0.06 & 28.5000211 \\
\hline 12 & & & & & & \\
\hline 13 & Load & Vert Disp. & Vert Disp. & Sine of Angle & Horz Disp. & Radius \\
\hline 14 & 0 & 0 & 0 & 0 & 0 & \#DIV/OI \\
\hline 15 & 2300 & 0.086 & 0.061 & 0.00131579 & 0.04 & 30.4000088 \\
\hline 16 & 4600 & 0.149 & 0.107 & 0.00221052 & 0.063 & 28.5000232 \\
\hline 17 & 7000 & 0.242 & 0.175 & 0.00352631 & 0.104 & 29.4925984 \\
\hline 18 & & & & & & \\
\hline 19 & Load & Vert Disp. & Vert Disp. & Sine of Angle & Horz Disp. & Radius \\
\hline 20 & 0 & 0 & 0 & 0 & 0 & \#DIV/O! \\
\hline 21 & 600 & 0.03 & 0.013 & 0.00089474 & 0.014 & 15.6470609 \\
\hline 22 & 1200 & 0.043 & 0.018 & 0.00131579 & 0.02 & 15.2000044 \\
\hline 23 & 1800 & 0.057 & 0.024 & 0.00173684 & 0.026 & 14.9697045 \\
\hline
\end{tabular}

\begin{tabular}{|c|c|c|c|}
\hline Load & Pad I Radius & Pad 3 Radius & Average Padus \\
\hline 0 & 0 & 0 & 0 \\
2300 & 26.703 & 30.400 & 28.551 \\
4600 & 25.017 & 28.500 & 26.7585 \\
7000 & 23.866 & 29.492 & 26.679 \\
\hline $60 a d$ & Pad Radus & Pad Radus & Average Padius \\
\hline 0 & 0 & 0 & 0 \\
1200 & 28.880 & 15.647 & 22.2635 \\
1800 & 28.500 & 14.969 & 21.735 \\
\hline
\end{tabular}


Gadle 2 (cont.) Appendit

$P$ ts $B$ and $C$

\begin{tabular}{|c|l|}
\hline & \multicolumn{1}{|c|}{$G$} \\
\hline 1 & Angle \\
\hline 2 & 0 \\
\hline 3 & 0.00194737 \\
\hline 4 & 0.00315789 \\
\hline 5 & 0.00431578 \\
\hline 6 & \\
\hline 7 & Angle (in rad) \\
\hline 8 & \\
\hline 9 & 0.00131579 \\
\hline 10 & 0.00189474 \\
\hline 11 & 0.00210526 \\
\hline 12 & \\
\hline 13 & Angle (in rad) \\
\hline 14 & \\
\hline 15 & 0.00131579 \\
\hline 16 & 0.00221053 \\
\hline 17 & 0.00352632 \\
\hline 18 & \\
\hline 19 & Angle (in rad) \\
\hline 20 & \\
\hline 21 & 0.00089474 \\
\hline 22 & 0.00131579 \\
\hline 23 & 0.00173684 \\
\hline & \\
\hline
\end{tabular}

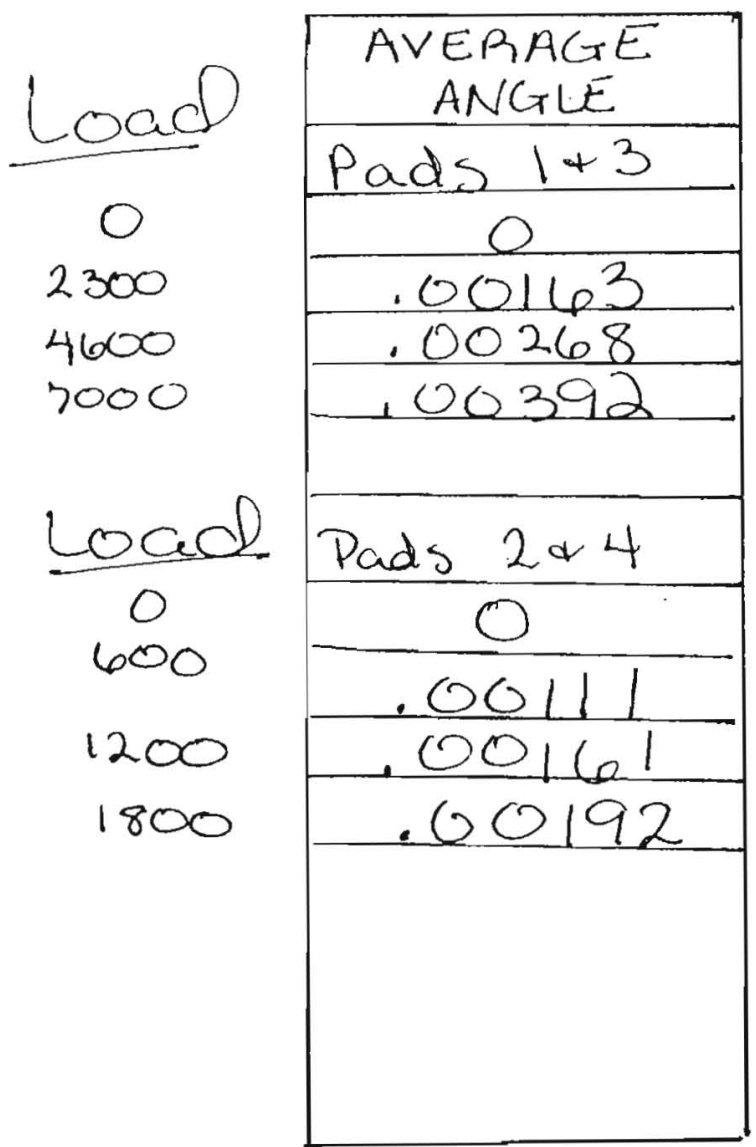




\section{Procedure for Testing the Structural Adequacy of the EC Cryostat Carriago}

1. Fit pinned extensions to the carriage.

1.1 Check mating of bearing surfaces.

1.2 Weld nuts (8) to carriage and fix extensions with bolts or studs.

2. Assemble test apparatus.

2.1 Use shims as necessary to level carriage.

2.2 Properly locate $12^{\prime \prime}$ diameter aluminum shims on upper plates of the carriage.

2.3 Place jacks on top of shims.

2.4 Lift lower beams and place on jacks.

2.5 Place 11.5" spacers atop beam assembly.

2.6 Lift upper beams and place on spacers.

2.7 Place four vertical links for operating load test. Make

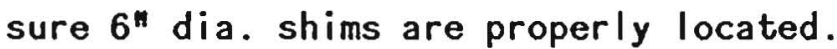
jacks to top of upper beam assembly.

2.8 Set up optical instruments and install rules for measuring deflections. 
3. Operating Load Test (Ref. 3740.220-MD-279338)

3.1 Record initial arm positions.

3.2 Simultaneously pressurize 150 ton jacks to 2300 psig., pressurize 100 ton jacks to 600 psig. Visually inspect apparatus for excessive distortions, misalignments, etc.

3.3 Record all deflection information.

3.4 Slowly increase the pressure in the jacks and take readings at 4600 psig (150T), 1200 psig (100T), and 7000 psig (150T), 1800 psig (100T)

3.5 Reduce the pressure in all of the jacks to zero and record readings.

3.6 Repressurize and record readings at 4600 psig (150T), 1200

3.7 Reduce pressure to zero and record readings.

3.8 Remove all optical equipment.

4. Lift Load Test (Ref. $3740-M D-279312$ )

4.1 Place lift test links at outer points.

4.2 Energize jacks and record pressure.

4.3 Connect links to pinned extensions and assure that links are vertical.

4.4 Slowly pressurize jacks to 2600 psig. (150T) and 670 psig. (100T), watching for signs of yielding, etc.

4.5 Slowly increase pressure in jacks and take readings at 5300 psig. (150T), 1300 psig. (100T) and 8000 psig. (150T) , 2000 psig. (100T).

4.6 Reduce pressure to zero

5. Disassemble apparatus and move pieces to long-term storage. Return equipment.

6. Remove pinned extensions and arrange for long-term storage. 
7. Place carriage on heavy duty carts and roll outside for storage. 
$1 / 7 \quad \operatorname{Kw} 3 / 90$

EC carriage test fixture

EMPTY WT. OF VESSEL (MODULES \& VESSELS):

$$
191,550^{\#} \text { (REAR) 31425 \# (FRONT) }
$$

LIFT TEST a 125\%:

$$
\text { M40,000 REAR 40,000 FRONT }
$$

WEIGHT WITH ARGON:

$$
320000^{\text {\# REAR } 36000 \text { F FRONT }}
$$

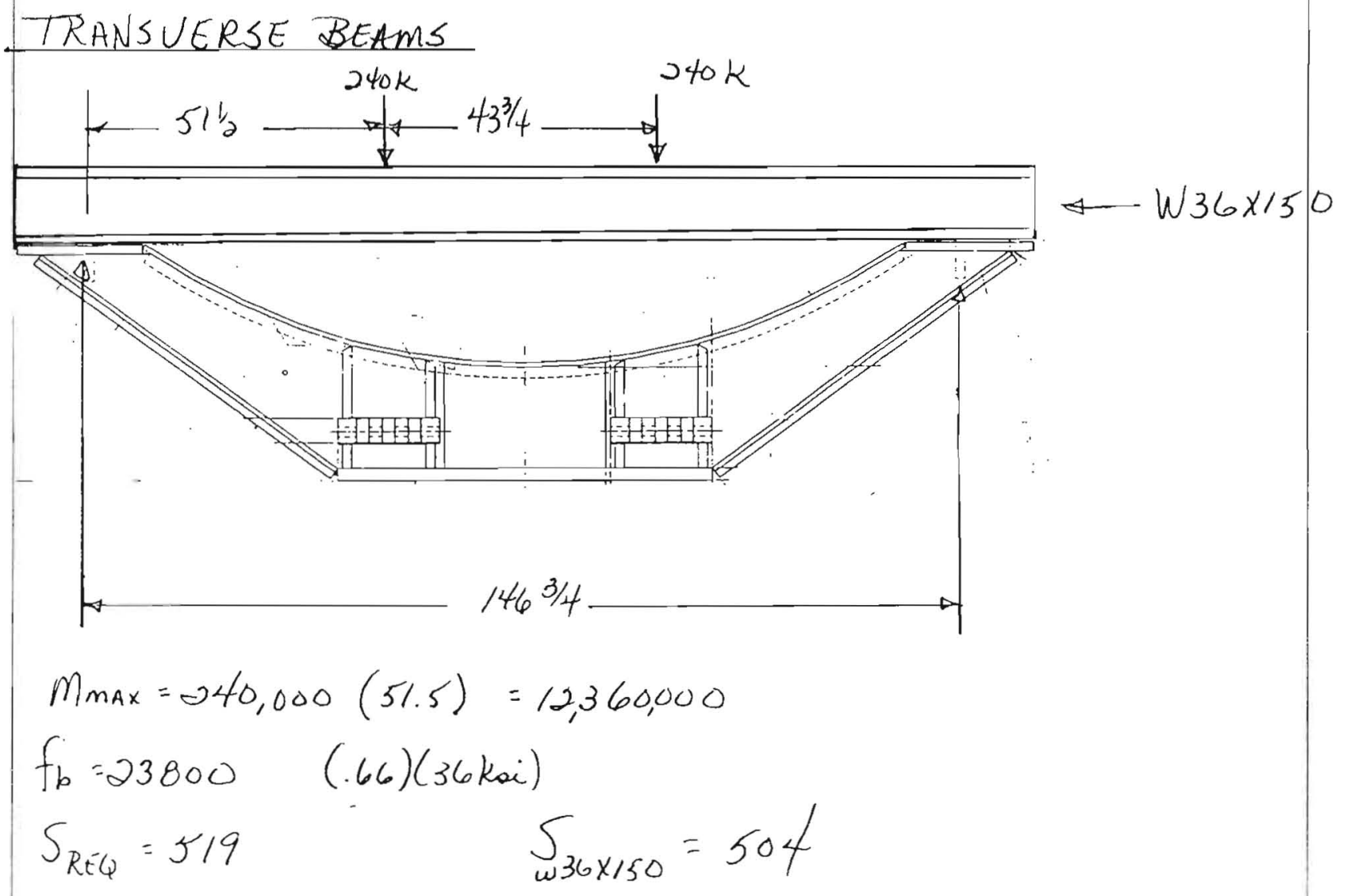


$2 / 1 \quad K \omega 3 / 90$

ADP PLATES ON TOP \& BOTTOM

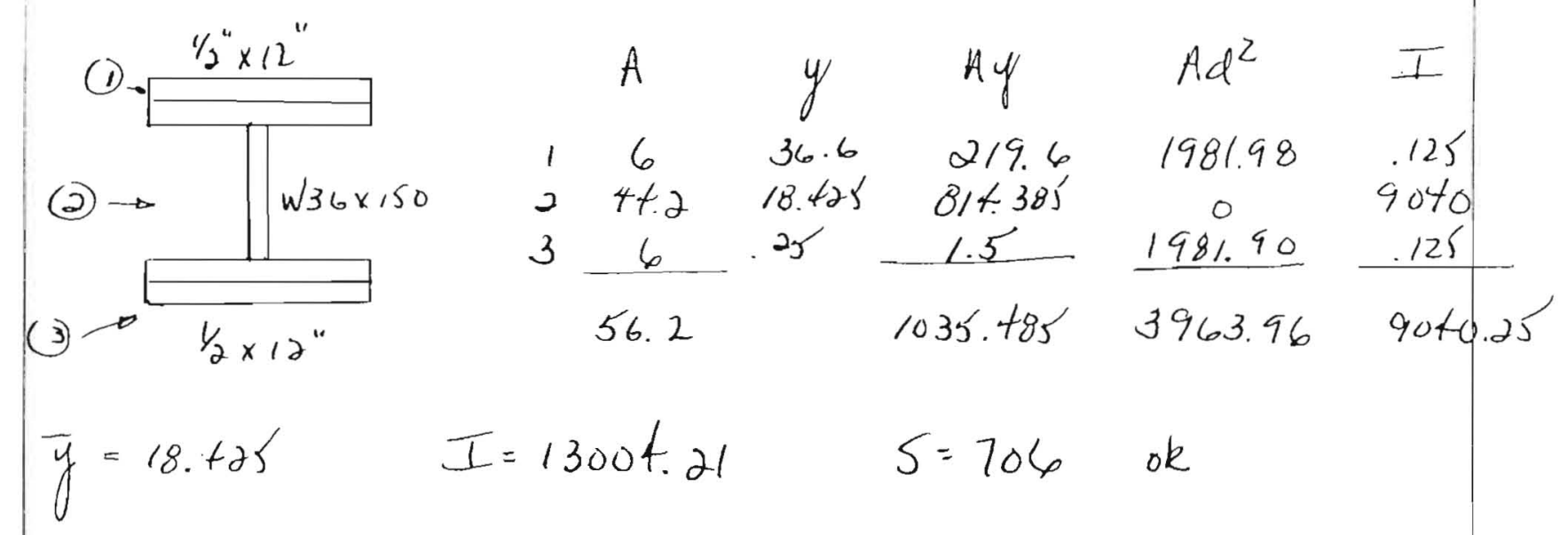

SHEAR

$$
\begin{aligned}
& F_{V}=.4(36)=14.4 \mathrm{ksi} \\
& A_{\min } \frac{240,000}{14400}=16.7 \mathrm{ok}
\end{aligned}
$$

WEB GRIPPING

USE $12 " \phi S H \mathrm{~mm}$

$$
\frac{240,000}{.625(12+2(1.875))}=04380<27,000 \quad(.75)(36)
$$


$3 / 7$ KW $3 / 9$

LONGITUDINAL BEAMS
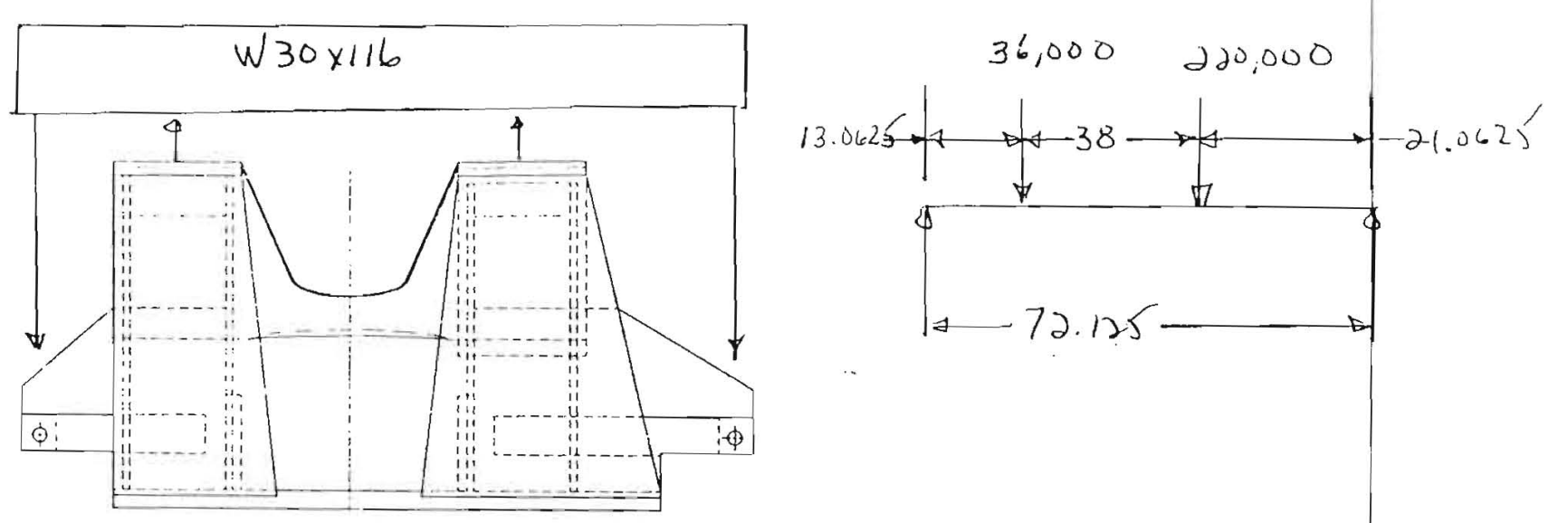

$$
\begin{aligned}
& R_{1}=\frac{36000(72.125-13.0625)+220,000(21.0625)}{72.125}=93726^{4} \\
& R_{2}=\frac{36000(13.0625)+220,000(72.125-21.0625)}{72.125}=162274^{1 t} \\
& M_{1}=93726(13.0625)=1224296 \\
& M_{2}=162274(21.0625)=3417892 \\
& S_{w_{30 \times 116}}=329 \quad \text { AREA }=16.9 \\
& V_{1}=\frac{122+296}{324}=3121 \quad V_{2}=\frac{3417892}{329}=10389
\end{aligned}
$$

SHEAR

$$
\frac{220,000}{16.9}=13017.75
$$


$4 / 1$ KW 3/90.

LONGITUPINAL BEAMS

$\omega 30 \times 116$
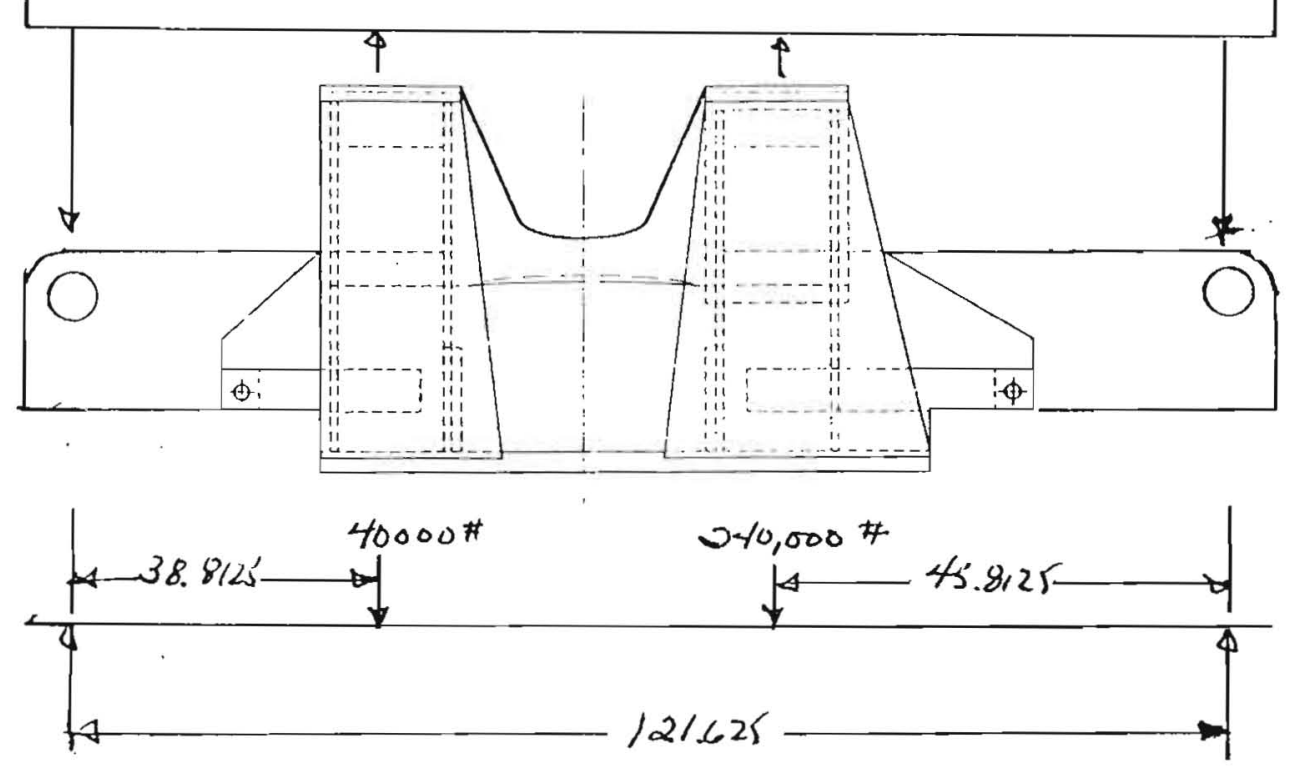

$$
\begin{aligned}
& R_{1}=\frac{40,000(84.8125)+240000(45.8125)}{121.625}=118294^{\#} \\
& R_{2}=\frac{40000(36.8125)+240000(75.8125)}{121.025}=161706^{\#} \\
& m_{1}=118294(36.8125)=4354698 \\
& m_{2}=161706(45.8125)=7408156 \quad \quad f_{b}=23800= \\
& V_{1}=13236 \quad V_{2}=22517 \quad
\end{aligned}
$$

$S=329$

$A=16.9$

SHEAR

$$
\frac{240000}{16.9}=14201 \quad F_{V}=.4(36)=14.4 \mathrm{kai}
$$


$5 / 7 \mathrm{kw} 3190$

VERTICAL MEMBERS

$$
\begin{aligned}
& F_{a}=2 / 600 \\
& A>\frac{240,000}{2(21600)}=5.56 \mathrm{in}^{2} \\
& 6 \times 6 \text { BARS } A=36 \\
& V=\frac{240,000}{36}=6667 \mathrm{ok} \\
& m_{\max }=\frac{240,000(24)}{4}=1440000 \\
& F_{b}=\frac{1440000}{36}=40,000 \\
& F_{p}=.75(50 \mathrm{Roi})=37,500
\end{aligned}
$$

PLATE

$$
F_{y}=12000
$$$$
F_{V}=8000
$$

SEC MON A

$$
\frac{P}{A}=\frac{240,000 / 2}{2 \times 5}=12000
$$

$$
s=36
$$

SECTION B

$$
f_{a}=\frac{240,000 / 4}{2.5 \times 2}=12000
$$

SECTION

$$
f_{v}=\frac{240000 / 2}{14}=8575
$$


6/7 $\mathrm{kw} \mathrm{3/90}$

VECTION D

$$
f_{p}=\frac{\partial 40,000 / 2}{\partial(b)}=10,000
$$

SECTION G PINNED EXIENSION

$$
f_{p}=\frac{240,000 / 2}{2(3,25)}=18461
$$

SECTION I

$$
f_{a}=\frac{240,000 / 2}{3(3)}=13,333
$$

SECTON E

$$
\begin{aligned}
e & =1.84375 \\
f_{a} & =\frac{240,000 / 2}{2(3)(1.84375)}=10,847
\end{aligned}
$$

SELTION F

$$
f_{v}=\frac{\partial 40,00 d(2)}{\partial(3)(3.5)}=5315
$$




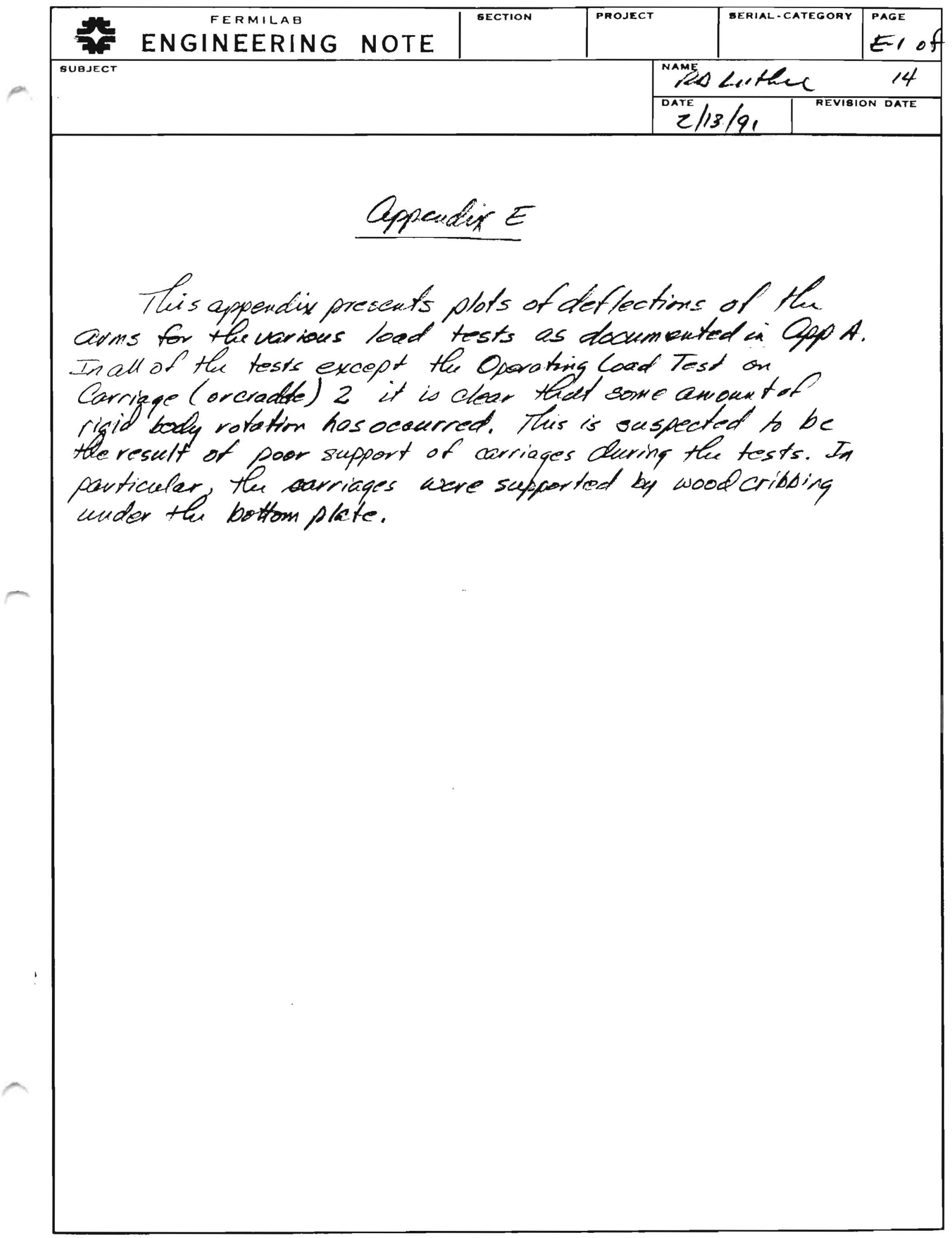




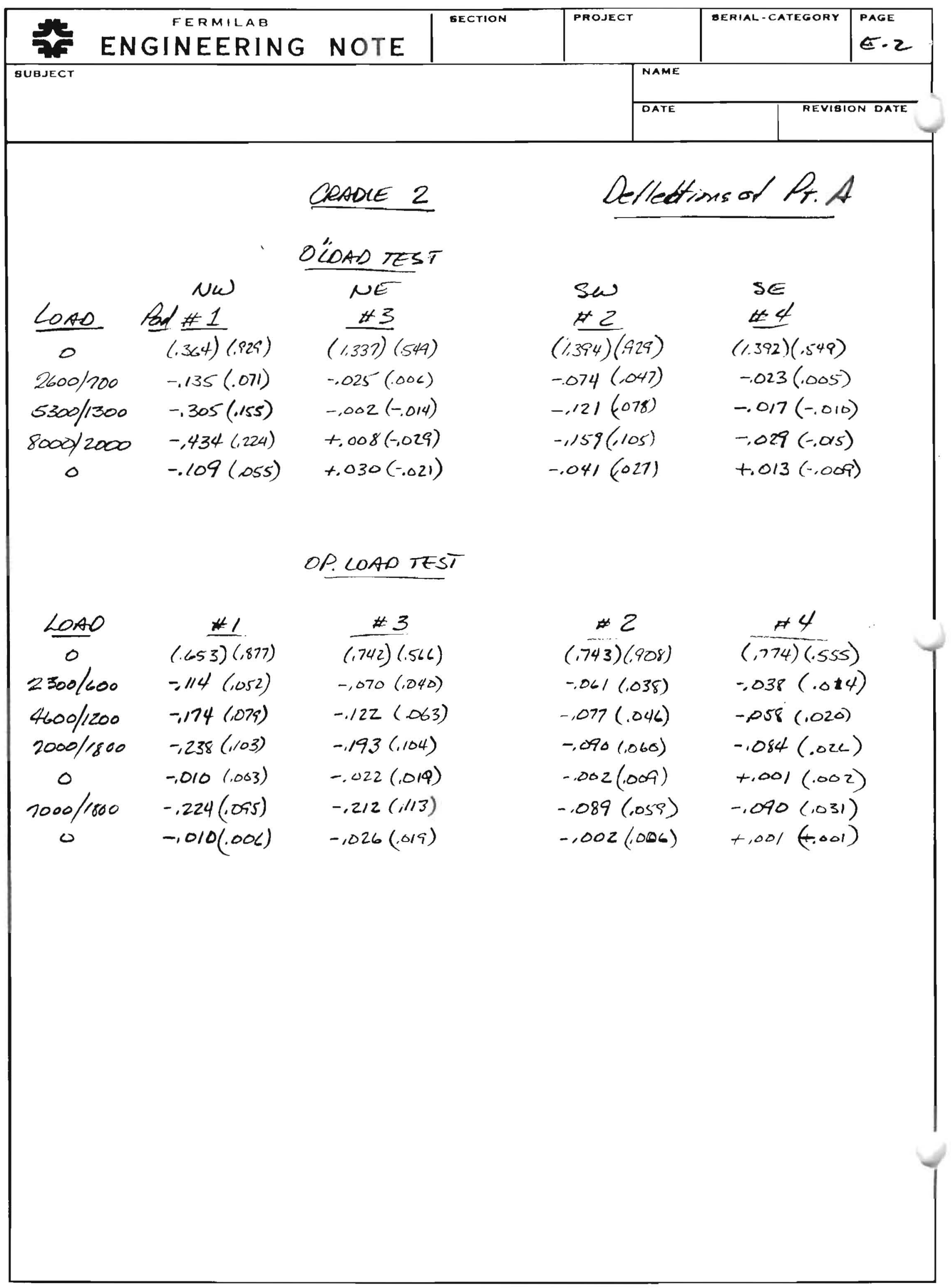




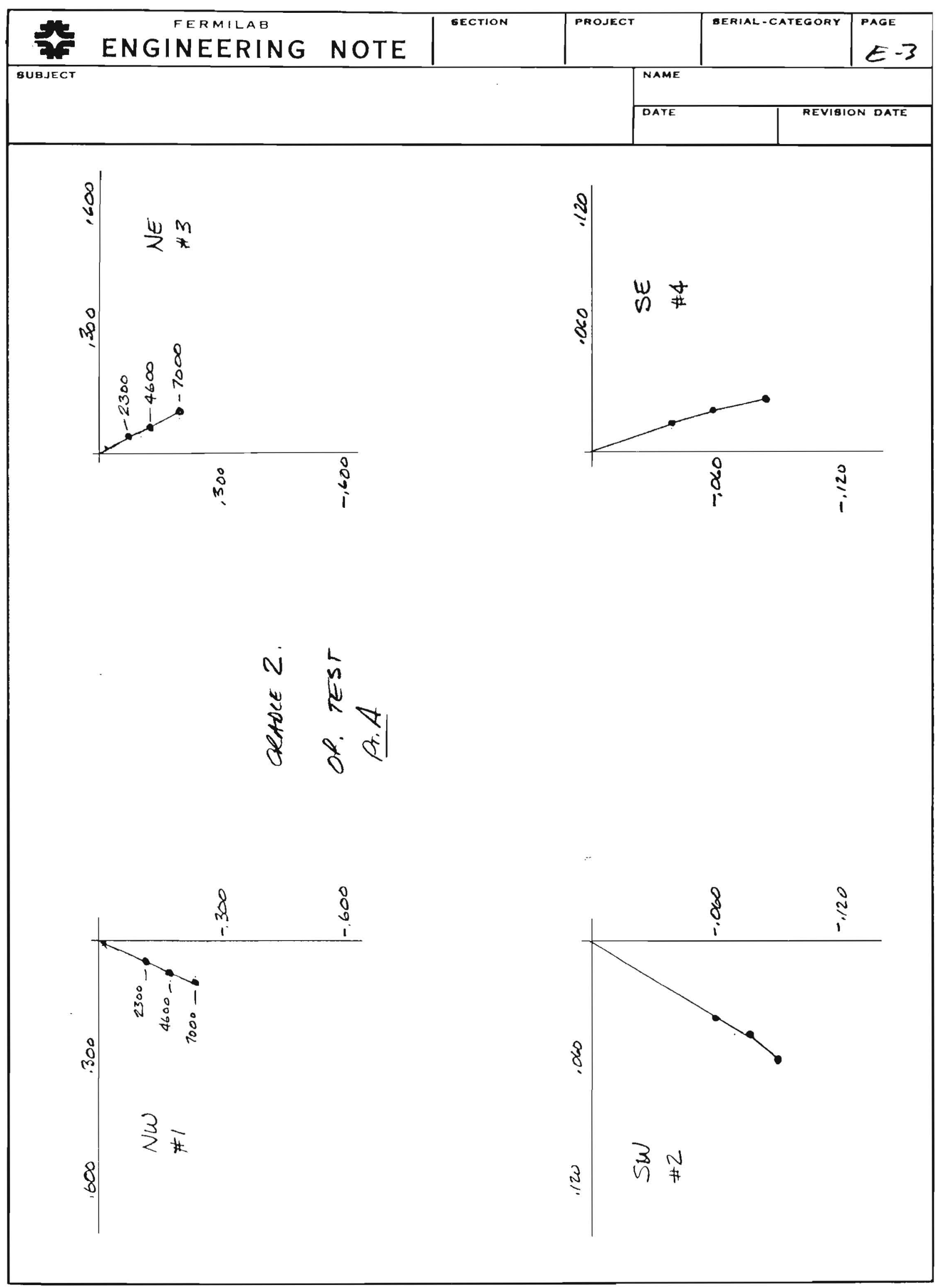




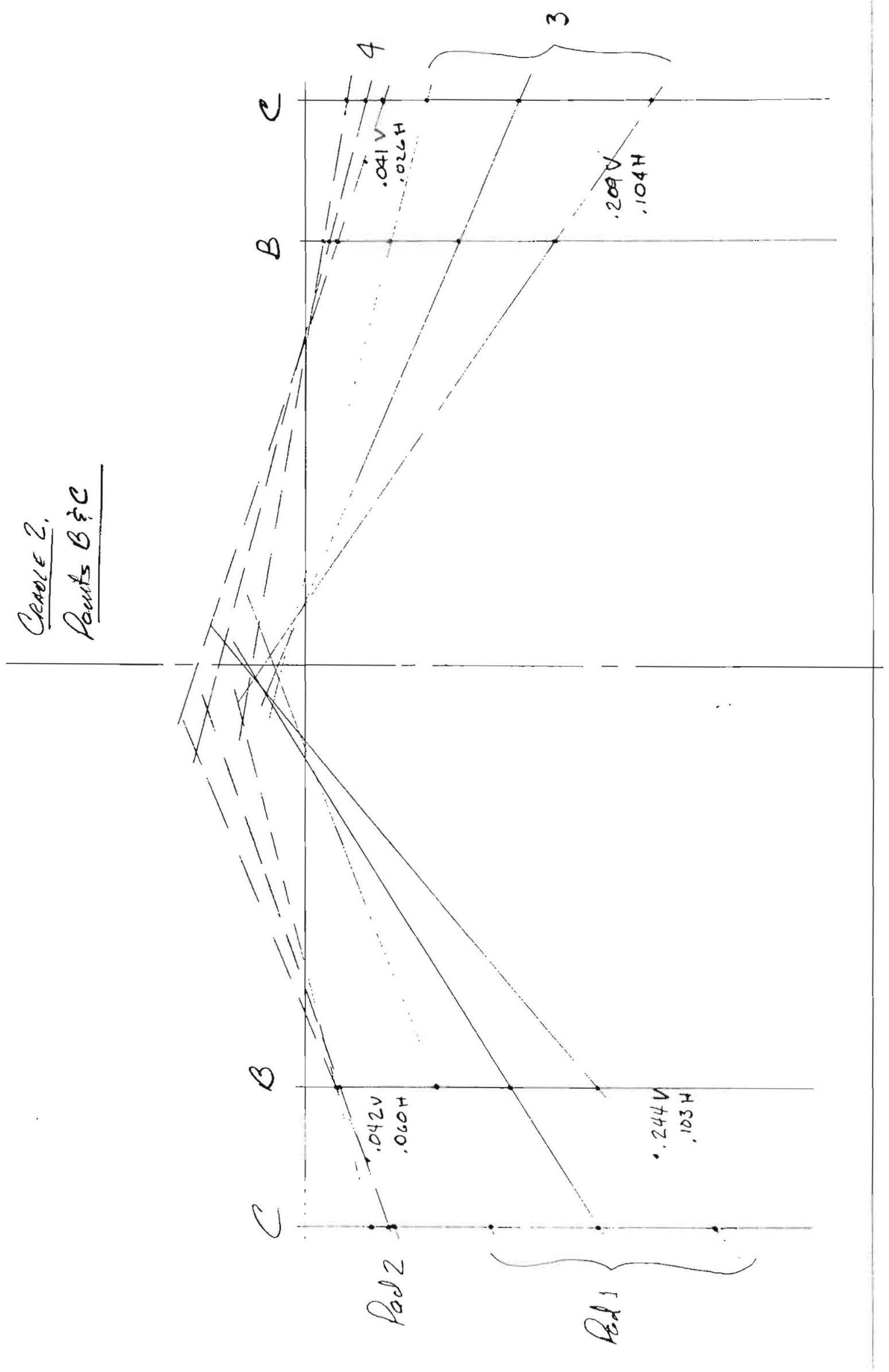




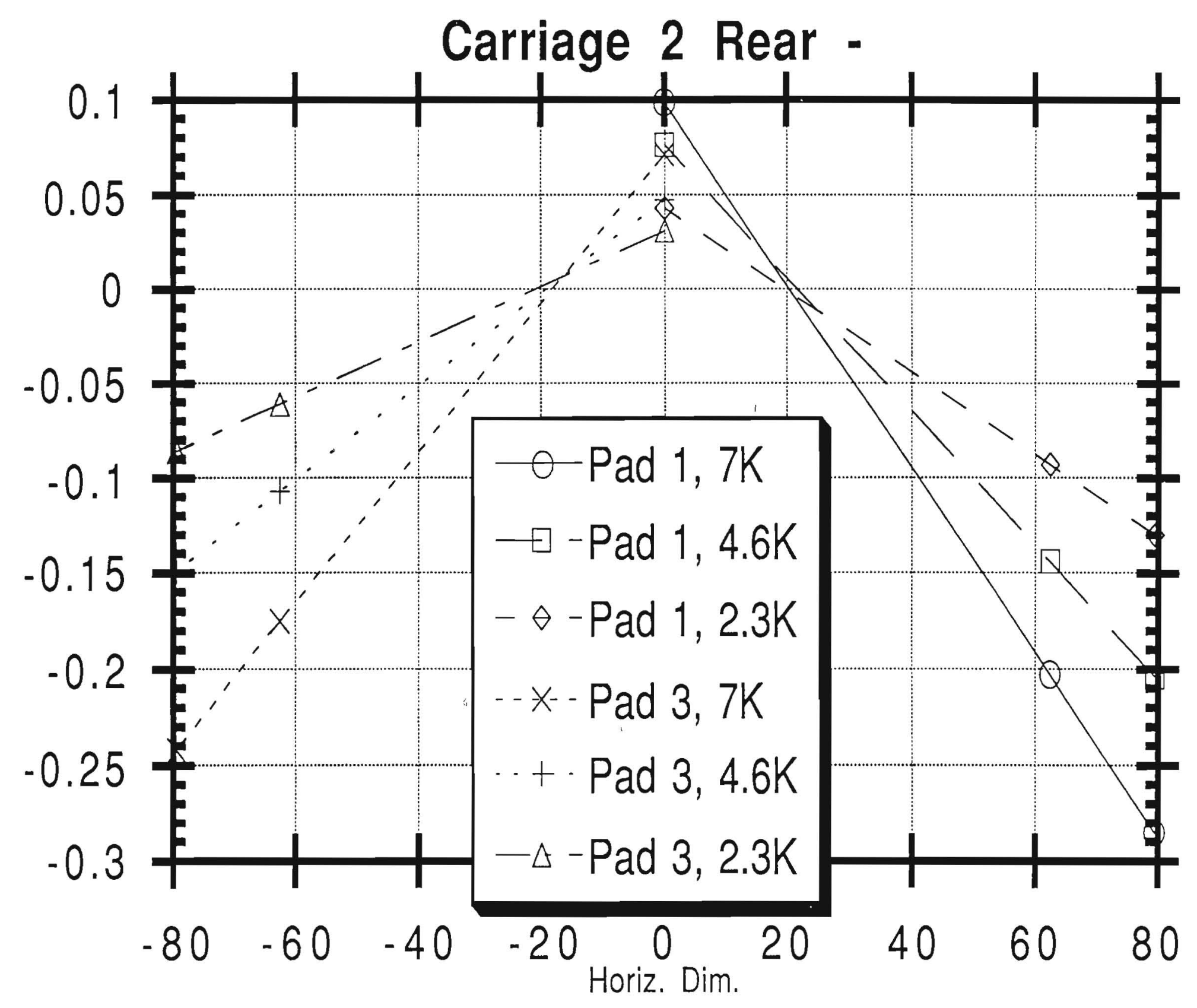




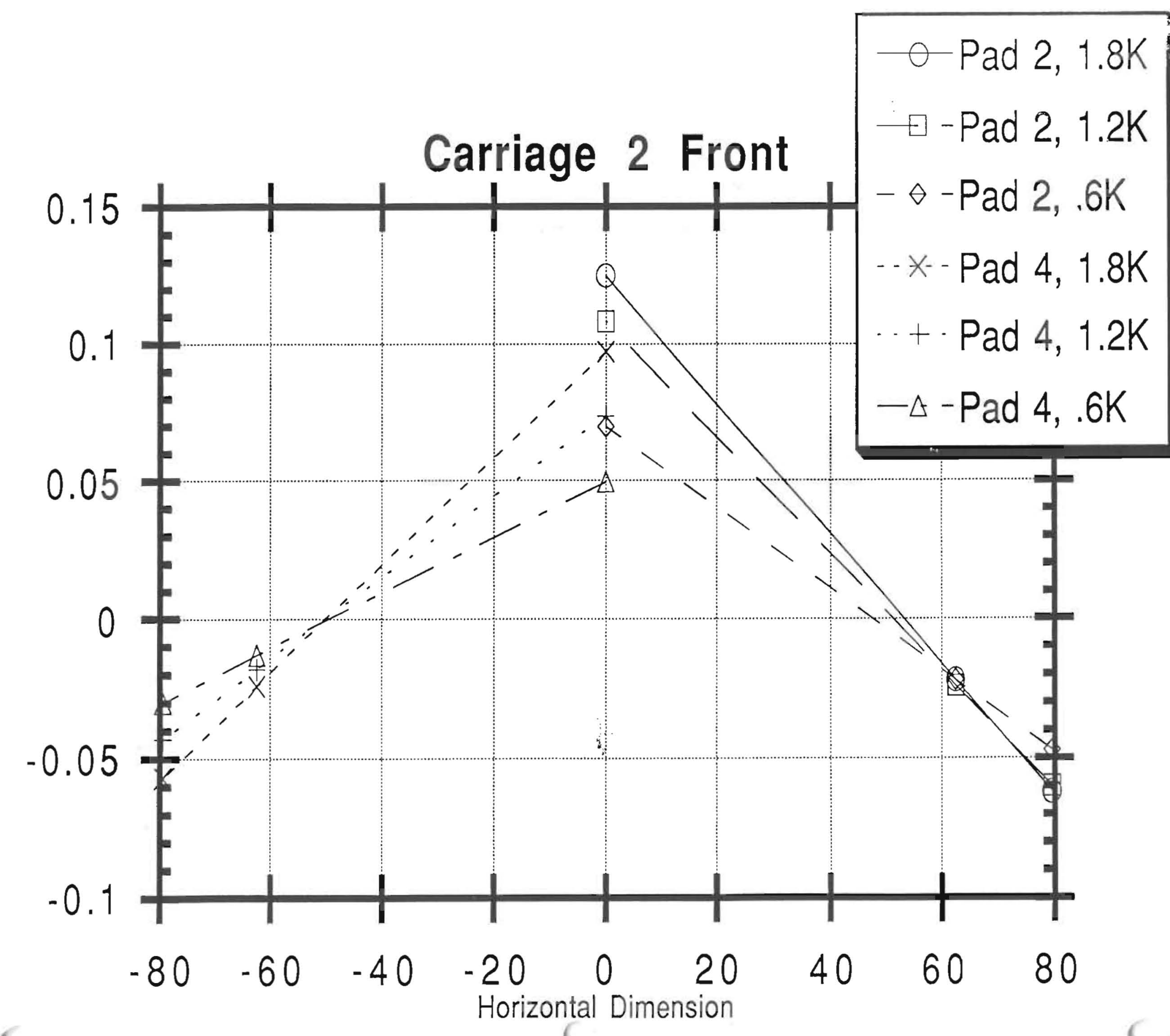




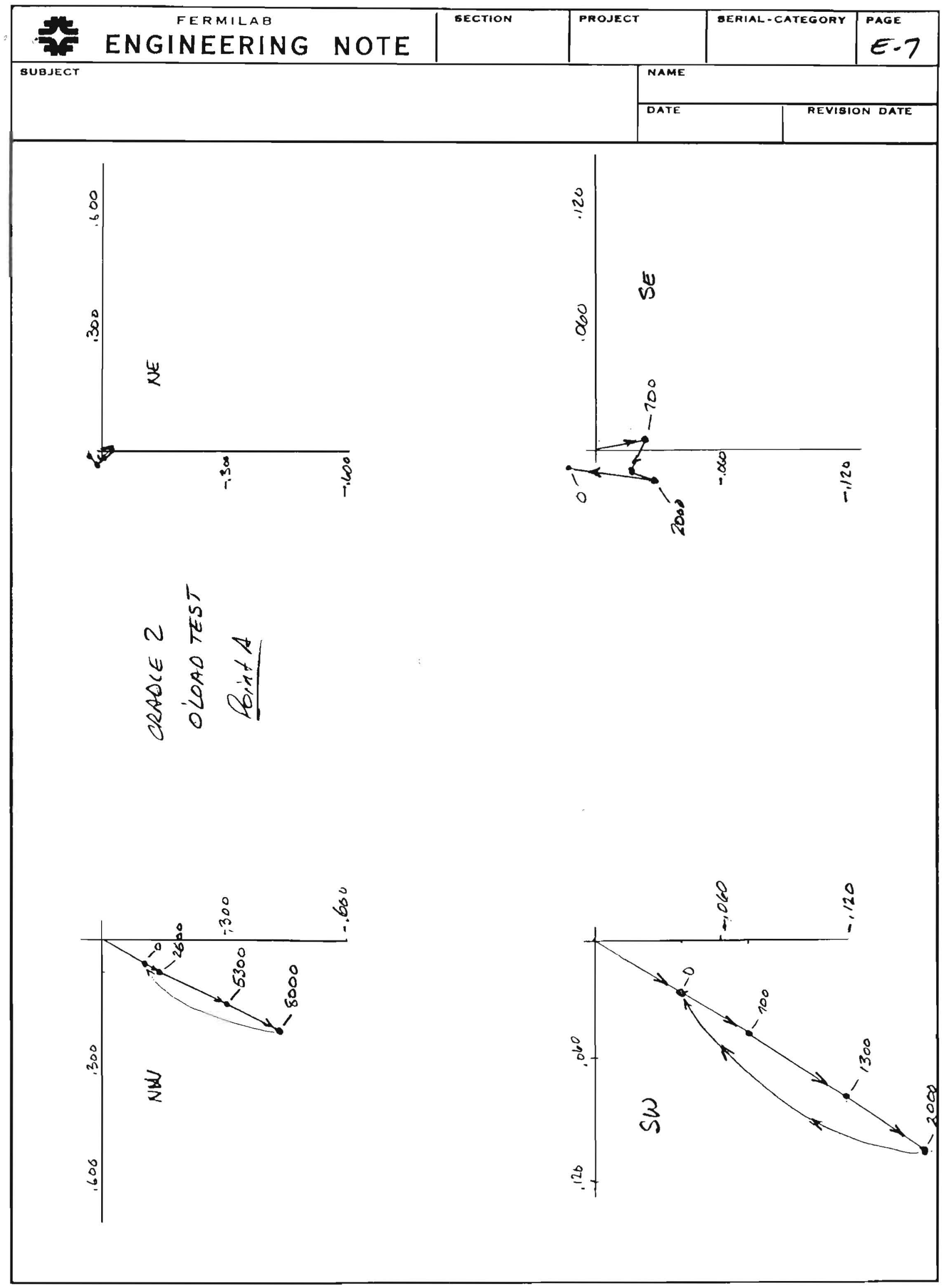




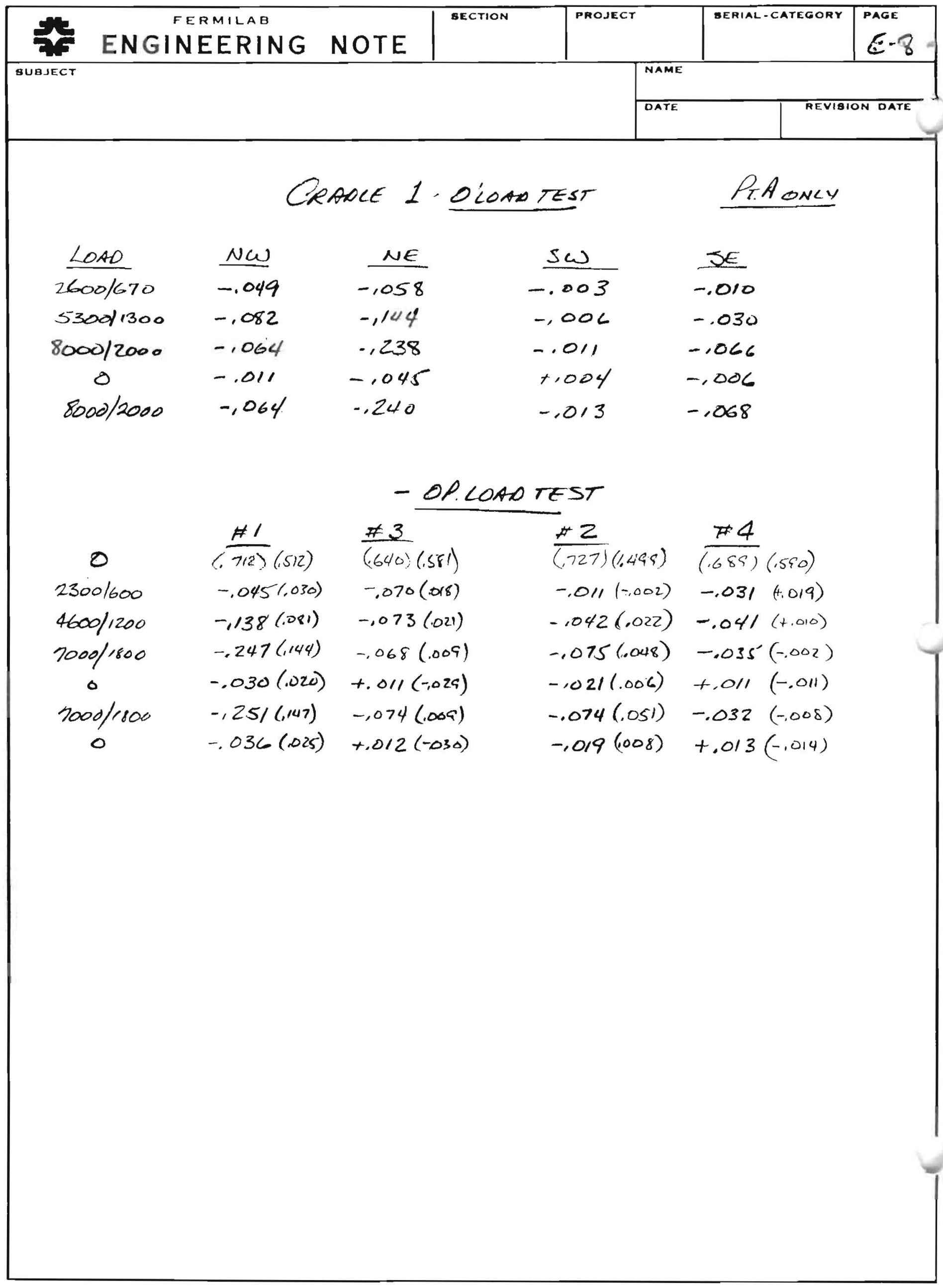




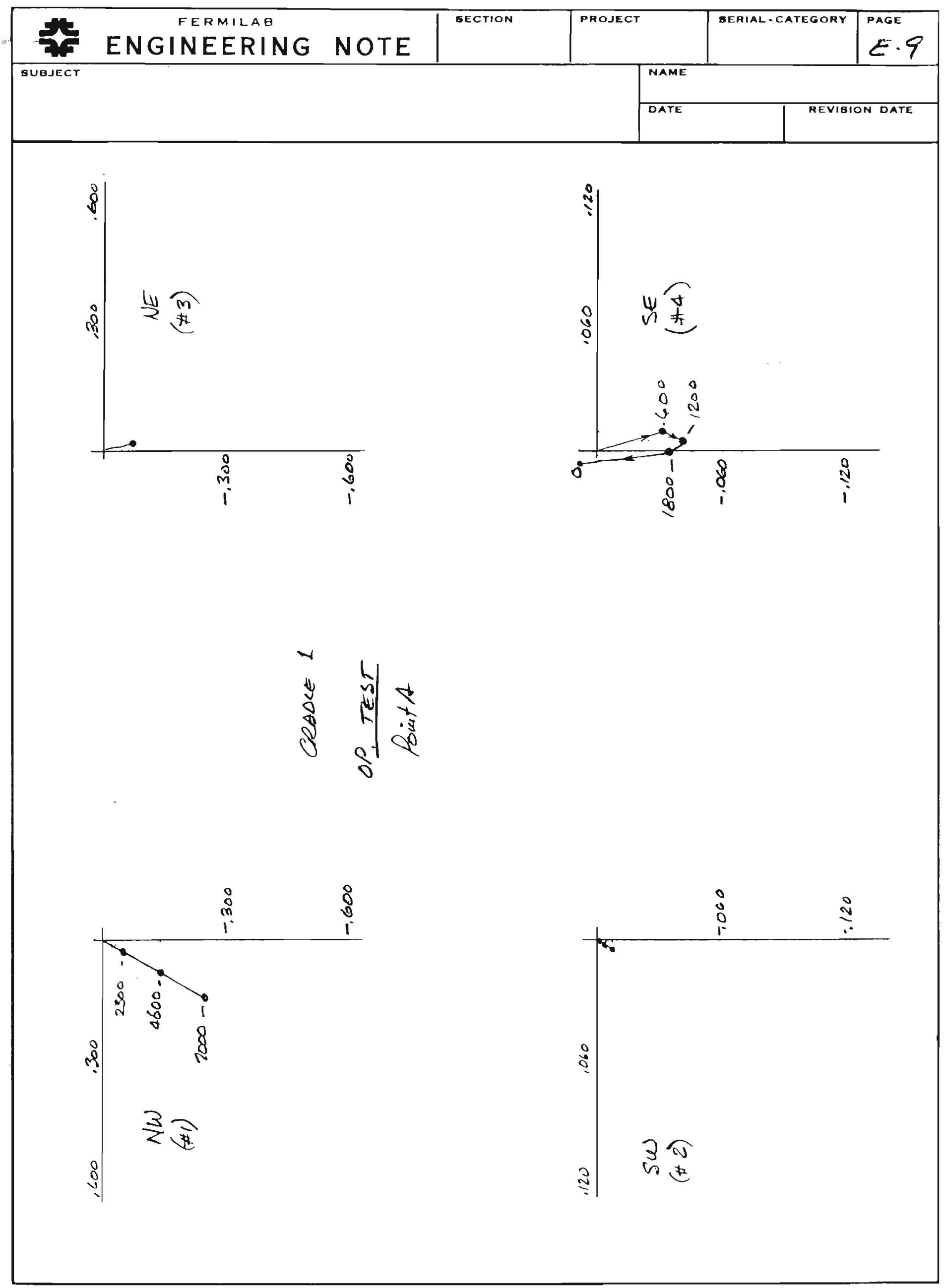




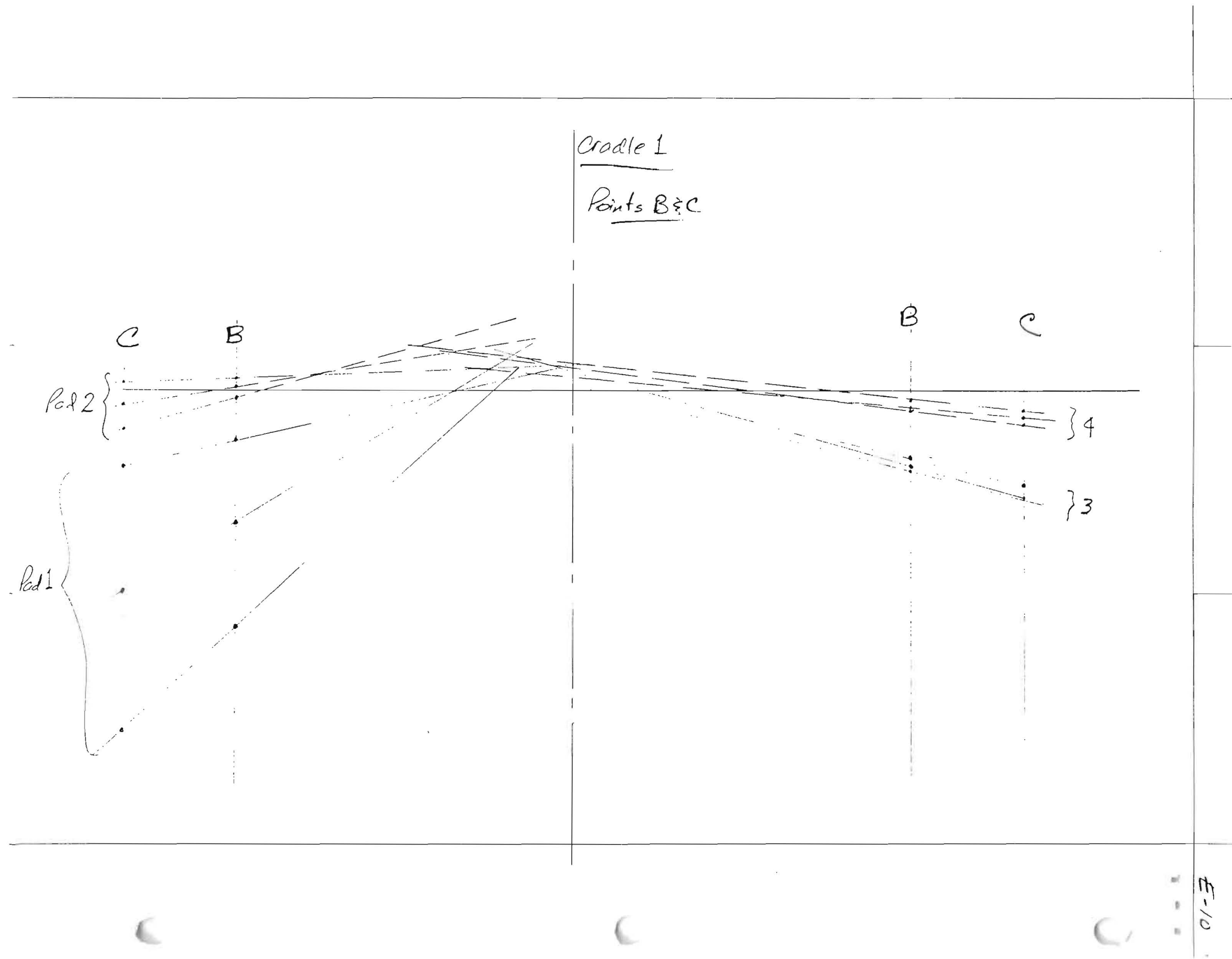




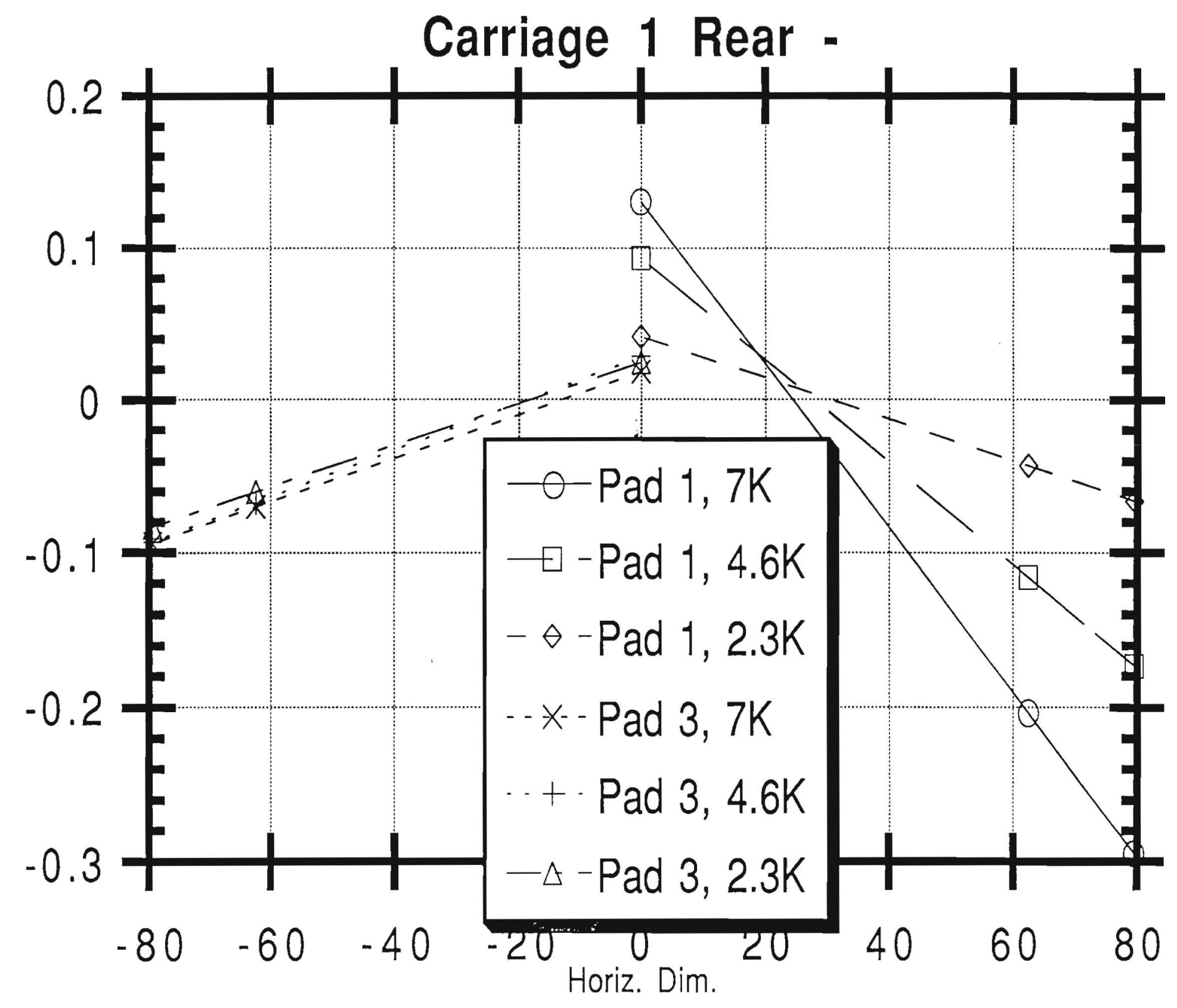




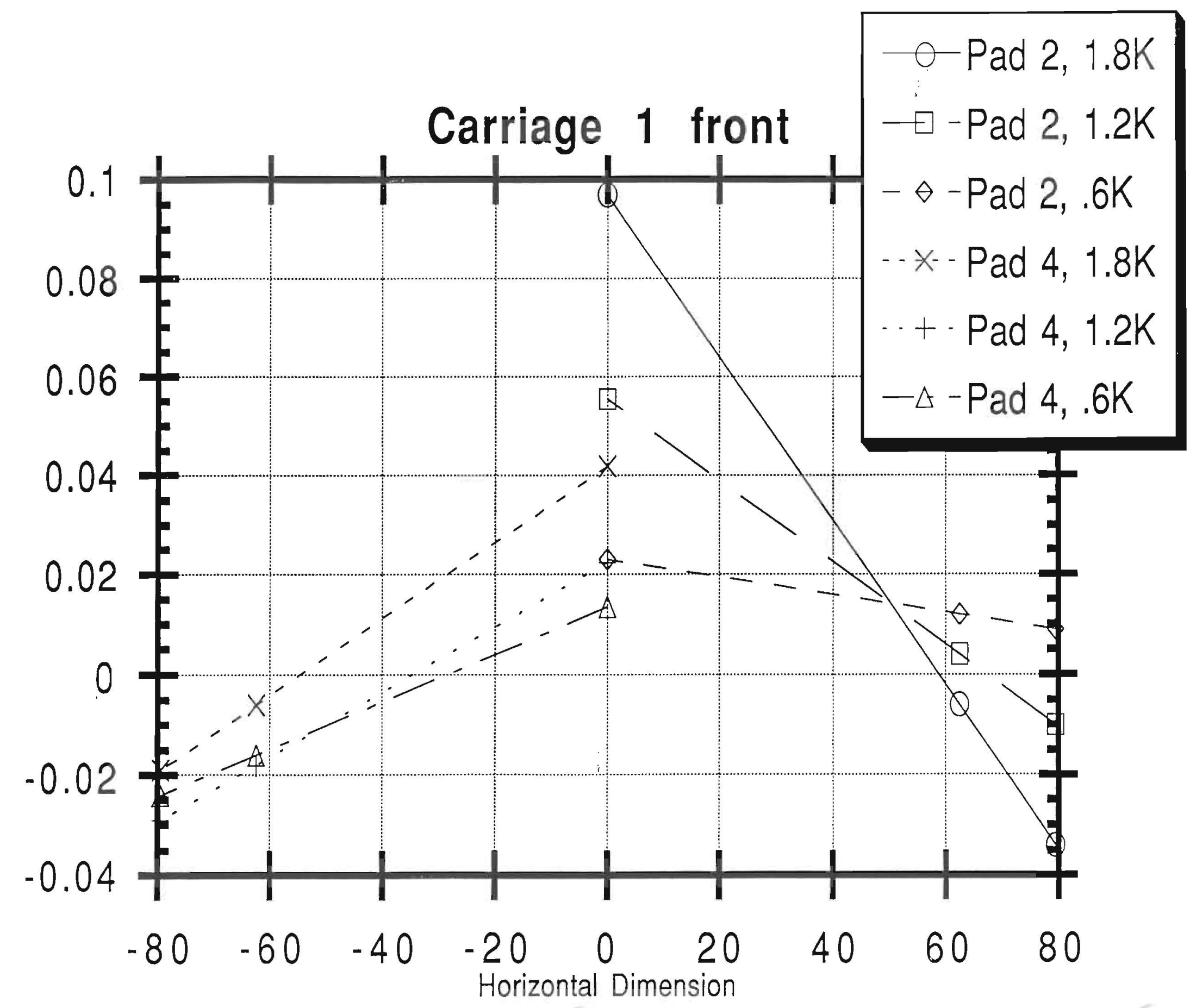




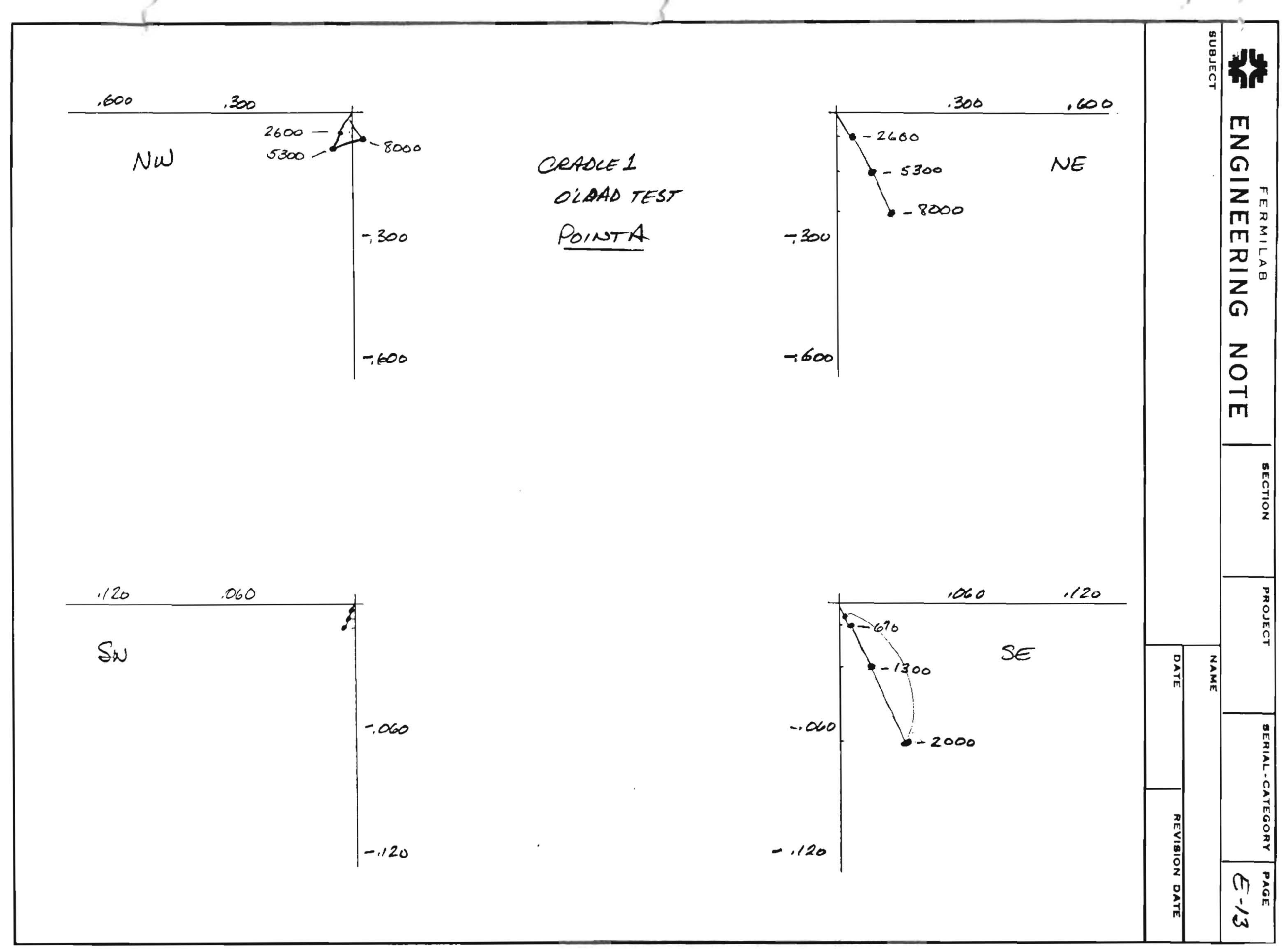




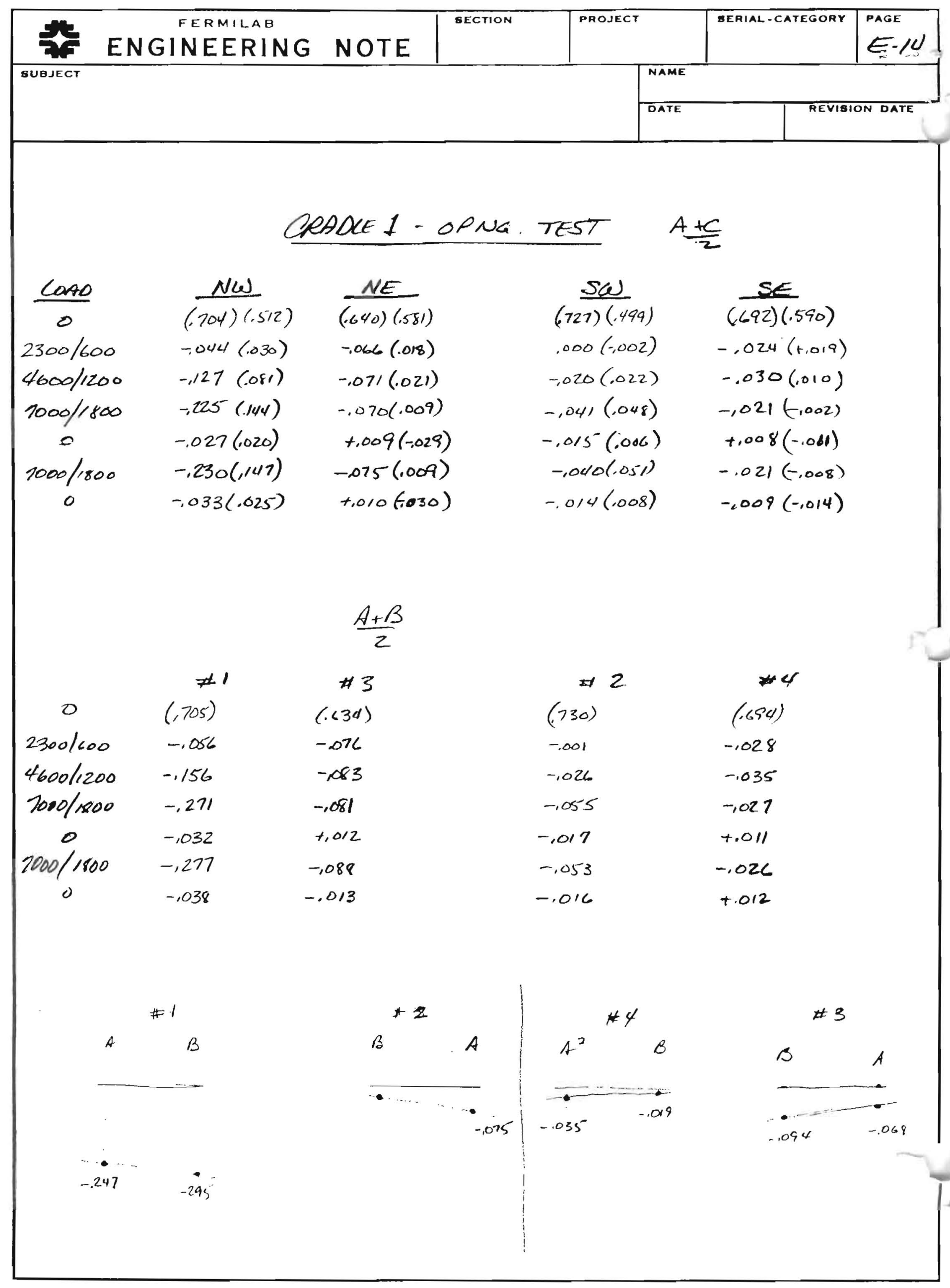

